\title{
REDUCTION OF LIE ALGEBROID STRUCTURES
}

\author{
J.F. CARIÑENA, J.M. NUNES DA COSTA AND PATRÍCIA SANTOS
}

\begin{abstract}
Based on the ideas of Marsden-Ratiu, a reduction method for Lie algebroids is developed in such a way that the canonical projection onto the reduced Lie algebroid is a homomorphism of Lie algebroids. A relation between Poisson reduction and Lie algebroid reduction is established. Reduction of Lie algebroids with symmetry is also studied using this method.
\end{abstract}

KEYWORDs: Lie algebroids, Poisson geometry, reduction, symmetry groups. AMS Subject Classification (2000): 17B80, 53D17, $70 \mathrm{H} 33$.

\section{Introduction}

Lie algebroids structures are very common in geometry, and are receiving an increasing interest in mathematics during the last years. The Lie algebroids were introduced by Pradines [24] as infinitesimal objects for differentiable groupoids and one can find similar notions proposed by several authors in increasing number of papers (which proves their importance and naturalness). For basic properties and the literature on the subject we refer to the survey paper and the book by Mackenzie [18, 19]. Weinstein [29] was the first pointing out the possibility of developing a Lagrangian mechanics in Lie algebroids (see also [17]) and then Martínez [21] developed a formalism for Lagrangian mechanics on Lie algebroids using the generalization of the fundamental ingredients of geometric Lagrangian mechanics. Afterwards several papers on related subjects are developed, see [16] and references therein. Roughly speaking, a Lie algebroid is a generalization of both a Lie algebra and of a tangent bundle, these being the simplest examples of Lie algebroids. A Lie algebroid is endowed with a differential operator playing a rôle very similar to the de Rham exterior differential. Furthermore, its dual has a natural Poisson structure. For these reasons Lie algebroids appear in many areas of mathematics, they have applications in mechanics and turn out to be a very import tool in the geometric foundations of physics. One of the most important examples of Lie algebroids with equal importance to physics

Received June 29, 2005.

The authors acknowledge the financial support from PRODEP/5.3/2003, POCTI/MAT/ 58452/2004, CMUC/FCT and the project BFM-2003-02532. 
is the gauge algebroid $T P / G$ associated to a principal bundle $P(M, G)$ [19]; remember that in classical field theory $M$ is the space-time manifold and $G$ is the gauge group, while matter fields are the sections of an associated bundle and the gauge fields describing matter interaction are connection 1-forms in $P(M, G)$.

The study of related structures in manifolds is very important because allows us to obtain at least a partial information on a system from properties of a related one, the theory of reduction being a particular example of this. In this way one can obtain, for instance, properties of the integral curves of a vector field from those of a related one, or of a presymplectic structure from the reduced symplectic one in the reduced space. The theory of reduction has many applications (see [20] and references therein) and has been shown to be extremely useful for a deep understanding of many physical theories including, among others, systems with symmetry, Poisson structures, stability theory, and integrable systems. The importance of reduction theory and the recent interest on Lie algebroids as a tool to work in many mechanical systems, suggests that it may be worthwhile to study Lie algebroid reduction. One of our initial motivations to study Lie algebroid reduction was the work of Meucci $[22,23]$ in which the periodic Toda system is obtained from a Poisson structure on a manifold $M$ with a specific reduction of a set of compatible Lie algebroid structures on a fibre bundle $A \rightarrow M$. The main purpose of this paper is to develop a general theory of reduction of Lie algebroids. We hope this reduction method could be adapted to other integrable systems of Toda and Calogero type. The reduction theory for Lie algebroids we develop here has been inspired by the techniques of Poisson reduction of Marsden and Ratiu [20] due to the strong connection between Poisson manifolds and Lie algebroids.

This paper is divided into five sections. In the section 2 we recall some well known facts about Lie algebroids, paying a special attention to the notion of homomorphism of Lie algebroids. Section 3 is devoted to the Lie algebroid reduction theory. Some examples are given and we also establish the conditions for the reduction of a Lie algebroid structure, and we prove that in these conditions the quotient bundle of a Lie algebroid is endowed with a differential operator that is a homological vector field of degree one; this operator defines on the quotient bundle a Lie algebroid structure which is the reduced structure of Lie algebroid. In section 4 we study the relation with Poisson reduction in the sense of Marsden-Ratiu, and we prove that the Lie 
algebroid reduction implies the Poisson reduction of its dual bundle in the sense of Marsden-Ratiu. Finally, in section 5, we study the representations of Lie groups in Lie algebroids and we show that the contragredient representation defines a Poisson action on the dual bundle. The theory is illustrated with some examples. Finally, in the last section we study the reduction of Lie algebroids with symmetry, in particular the gauge algebroid as an example of reduced Lie algebroid.

\section{Basic concepts on Lie algebroids}

A Lie algebroid is a vector bundle $p: A \rightarrow M$ over a manifold $M$ together with a vector bundle morphism $\rho: A \rightarrow T M$ over the identity map on $M$ (called anchor) and a Lie bracket $[\cdot, \cdot]_{A}$ on the linear space $\Gamma(A)$ of sections for $p$ satisfying

$$
[v, f w]_{A}=f[v, w]_{A}+(\rho(v) f) w
$$

for every pair of sections $v$ and $w$ and any smooth function $f$ on $M$. We denote the Lie algebroid by $\left(A, \rho,[\cdot, \cdot]_{A}\right)$ or simply by $A$ whenever it is clear which Lie algebroid we refer to. Note that $\Gamma(A)$ is a $C^{\infty}(M)$-module and the anchor is a $C^{\infty}(M)$-linear map from the space $\Gamma(A)$ into the space $\mathfrak{X}(M)$ of vector fields on $M$, and one can easily prove, using the above condition and the Jacobi identity of the Lie bracket $[\cdot, \cdot]_{A}$, that the anchor is a Lie algebra homomorphism [13]. For a detailed lecture on the subject see e.g. [1, 19].

Two simple examples of Lie algebroids are the tangent bundle $\tau_{M}: T M \rightarrow$ $M$, with the identity as anchor map and the usual bracket of vector fields on $M$, and the Lie algebra $\mathfrak{g}$ when considered as a vector bundle over a point, $p: \mathfrak{g} \rightarrow\{\cdot\}$, with the anchor being the zero map; the sections of such bundle are but the elements of $\mathfrak{g}$ and the bracket of sections is the bracket of the Lie algebra. Two other examples to be used in this paper are the Lie algebroid associated with a Poisson manifold and the Lie algebroid provided by the action of a Lie group on a manifold, defined as follows (see e.g. [5]).

Example 2.1 (Poisson manifold). Let $(M, \Lambda)$ be a Poisson manifold, that is, $M$ is a manifold endowed with a bivector field $\Lambda$ such that $[\Lambda, \Lambda]_{S}=0$, where $[\cdot, \cdot]_{S}$ is the Schouten-Nijenhuis bracket [14]. This bivector field allows us to introduce a Poisson bracket in the set $C^{\infty}(M)$ by $\{f, g\}:=\Lambda(d f, d g)$ for all $f, g \in C^{\infty}(M)$. The contraction with the bivector field $\Lambda$ induces a vector bundle morphism $\Lambda^{\sharp}$ over the identity map on $M, \Lambda^{\sharp}: T^{*} M \rightarrow T M$, and therefore between the spaces of sections of both bundles, to be denoted by 
the same symbol $\Lambda^{\sharp}: \Omega^{1}(M) \rightarrow \mathfrak{X}(M),\left\langle\beta, \Lambda^{\sharp}(\alpha)\right\rangle=\Lambda(\alpha, \beta)$ for all $\alpha, \beta \in$ $\Omega^{1}(M)$. The cotangent bundle $T^{*} M$ can be endowed with a Lie algebroid structure whose anchor map is $\Lambda^{\sharp}$ and the Lie bracket of 1-forms on $M$ is given by $[\alpha, \beta]_{T^{*} M}=£_{\Lambda^{\sharp}(\alpha)} \beta-£_{\Lambda^{\sharp}(\beta)} \alpha-d(\Lambda(\alpha, \beta))$. Note that for exact 1-forms the bracket reduces to $[d f, d g]_{T^{*} M}=d\{f, g\}$ for all $f, g \in C^{\infty}(M)$. We call this Lie algebroid the Lie algebroid of the Poisson manifold.

Example 2.2 (Group action). Let $\phi: M \times G \rightarrow M$ be a right action of a Lie group $G$ on the manifold $M$ and for each element $X$ of the Lie algebra $\mathfrak{g}$ of $G$, let $X_{M}$ denote the corresponding fundamental vector field defined by $X_{M}(m)=\left.\frac{d}{d t} \phi(m, \exp (t X))\right|_{t=0}$ for all $m \in M$. The set $\Gamma(M \times \mathfrak{g})$ of sections of the trivial bundle $M \times \mathfrak{g} \rightarrow M$ can be identified with the space $C^{\infty}(M, \mathfrak{g})$ of smooth functions on $M$ with values in $\mathfrak{g}$ : each section $Y \in \Gamma(M \times \mathfrak{g})$ is of the form $Y(x)=(x, \tilde{Y}(x))$ with $\widetilde{Y} \in C^{\infty}(M, \mathfrak{g})$, for all $x \in M$. Then, the trivial bundle $M \times \mathfrak{g} \rightarrow M$ can be endowed with a Lie algebroid structure: we define a bracket on the space $\Gamma(M \times \mathfrak{g}) \cong C^{\infty}(M, \mathfrak{g})$ by setting

$$
\left[Y_{1}, Y_{2}\right]_{M \times \mathfrak{g}}(x)=\left(x,\left[\widetilde{Y}_{1}(x), \widetilde{Y}_{2}(x)\right]_{\mathfrak{g}}+\left(\widetilde{Y}_{1}(x)\right)_{M} \widetilde{Y}_{2}(x)-\left(\widetilde{Y}_{2}(x)\right)_{M} \widetilde{Y}_{1}(x)\right),
$$

for all $x \in M$ and $Y_{1}, Y_{2} \in \Gamma(M \times \mathfrak{g})$, which is a Lie bracket, and we define $\rho: M \times \mathfrak{g} \rightarrow T M, \rho(x, Y)=Y_{M}(x)$, as anchor map [21].

Given a Lie algebroid $\left(A, \rho,[\cdot, \cdot]_{A}\right)$, there exists a derivation $d_{\rho}$ of degree one of the graded exterior algebra of forms of $A, \Omega^{\bullet}(A)$, to be called $A$-forms, which is nilpotent of order two, i.e. $d_{\rho}^{2}=0$. It is called the exterior derivative of the Lie algebroid. In the particular cases of the tangent bundle $T M$ and that of a Lie algebra $\mathfrak{g}, d_{\rho}$ reduces to the de Rham operator on the manifold $M$ and the Chevalley differential, respectively [6].

On the other hand, the dual bundle $\tau: A^{*} \rightarrow M$ of a Lie algebroid $\left(A, \rho,[\cdot, \cdot]_{A}\right)$ over $M$ is endowed with a natural linear Poisson structure $\{\cdot, \cdot\}_{A^{*}}$ given by the fundamental brackets [7]:

$\{f \circ \tau, g \circ \tau\}_{A^{*}}=0,\{\chi(v), f \circ \tau\}_{A^{*}}=\rho(v) f \circ \tau,\{\chi(v), \chi(w)\}_{A^{*}}=\chi\left([v, w]_{A}\right)$, for all $f, g \in C^{\infty}(M)$ and $v, w \in \Gamma(A)$, where $\chi$ maps each section $v$ of $A$ to the linear function $\chi(v)$ given by $\chi(v)(\alpha)=\langle\alpha, v\rangle$ for all $\alpha \in \Gamma\left(A^{*}\right)$; here $\langle\cdot, \cdot\rangle$ denotes duality pairing between $\Gamma\left(A^{*}\right)$ and $\Gamma(A)$. The bivector field associated to such a Poisson bracket $\{\cdot, \cdot\}_{A^{*}}$ will be denoted $\Lambda_{A^{*}}$. We call Lie co-algebroid to the dual bundle of a Lie algebroid endowed with such linear Poisson structure. In particular, the dual $\mathfrak{g}^{*}$ of a Lie algebra $\mathfrak{g}$ has a natural 
Poisson structure, usually called the Lie-Poisson structure, while the Poisson structure on the cotangent bundle $T^{*} M$ to a manifold $M$ as dual bundle of the Lie algebroid $T M$ turns out to be the canonical symplectic structure.

2.1. Homomorphism of Lie algebroids. Let $(\Phi, \phi):(A, p, M) \rightarrow$ $\left(A^{\prime}, p^{\prime}, M^{\prime}\right)$ be a morphism of vector bundles. If $\phi=\operatorname{id}_{M}$ or if $\phi$ is a diffeomorphism, it induces a linear map between the spaces of sections: $v \in \Gamma(A)$ maps into $v^{\prime}=\Phi \circ v \in \Gamma\left(A^{\prime}\right)$ when $\phi=\mathrm{id}_{M}$ and maps into $v^{\prime}=\Phi \circ v \circ \phi^{-1} \in \Gamma\left(A^{\prime}\right)$ when $\phi$ is an arbitrary diffeomorphism. In the general case, however, we cannot define such morphism between the space of sections. Nevertheless, there is a vector bundle morphism $\bar{\Phi}: A \rightarrow \phi^{!} A^{\prime}$ over the identity in $M$, where $\phi^{!} A^{\prime}$ denotes the pull-back bundle [28], given by

$$
\bar{\Phi}_{x}: v_{x} \in A_{x} \rightarrow\left(x, \Phi_{x}\left(v_{x}\right)\right) \in\left(\phi^{!} A^{\prime}\right)_{x}
$$

for all $x \in M$. Recall that a section of $A^{\prime}$ along the map $\phi: M \rightarrow M^{\prime}$ is a map $V: M \rightarrow A^{\prime}$ such that $p^{\prime} \circ V=\phi$ and that the space $\Gamma_{\phi}\left(A^{\prime}\right)$ of sections of $A^{\prime}$ along the map $\phi: M \rightarrow M^{\prime}$ is a $C^{\infty}(M)$-module isomorphic to $\Gamma\left(\phi^{!} A^{\prime}\right)$. In particular, if $v \in \Gamma(A)$ then $\Phi \circ v \in \Gamma_{\phi}\left(A^{\prime}\right)$ and when $v^{\prime} \in \Gamma\left(A^{\prime}\right)$ then $v^{\prime} \circ \phi \in \Gamma_{\phi}\left(A^{\prime}\right)$. The $C^{\infty}(M)$-module of sections $\Gamma_{\phi}\left(A^{\prime}\right)$ is (locally) generated by the set of sections of the form $v^{\prime} \circ \phi$, with $v^{\prime} \in \Gamma\left(A^{\prime}\right)$, and then for any section $v \in \Gamma(A)$ we can write

$$
\Phi \circ v=\sum_{i} f_{i}\left(v_{i}^{\prime} \circ \phi\right)
$$

which is called a $\Phi$-decomposition of $v$.

The section $v \in \Gamma(A)$ is said to be $\Phi$-related with $v^{\prime} \in \Gamma\left(A^{\prime}\right)$, and we write $v \sim v^{\prime}$, when $\Phi \circ v=v^{\prime} \circ \phi$ and $v \in \Gamma(A)$ is called $\Phi$-projectable when there exists a $\Phi$-related section $v^{\prime} \in \Gamma\left(A^{\prime}\right)$. The set of such $\Phi$-projectable sections is denoted by $\Gamma^{\Phi}(A)$. Higgins and Mackenzie established in [10] the following definition of homomorphism of Lie algebroids:

Definition 2.1. A vector bundle morphism $(\Phi, \phi):(A, p, M) \rightarrow\left(A^{\prime}, p^{\prime}, M^{\prime}\right)$ is a homomorphism of the Lie algebroid $\left(A, \rho,[\cdot, \cdot]_{A}\right)$ over $M$ in the Lie algebroid $\left(A^{\prime}, \rho^{\prime},[\cdot, \cdot]_{A^{\prime}}\right)$ over $M^{\prime}$ when $T \phi \circ \rho=\rho^{\prime} \circ \Phi$ and, for any pair $v, w \in \Gamma(A)$ with $\Phi$-decompositions $\Phi \circ v=\sum_{i} f_{i}\left(v_{i}^{\prime} \circ \phi\right)$ and $\Phi \circ w=\sum_{j} g_{j}\left(w_{j}^{\prime} \circ \phi\right)$, the 
following condition is satisfied:

$\Phi \circ[v, w]_{A}=\sum_{i, j} f_{i} g_{j}\left(\left[v_{i}^{\prime}, w_{j}^{\prime}\right]_{A^{\prime}} \circ \phi\right)+\sum_{j}\left(\rho(v) g_{j}\right)\left(w_{j}^{\prime} \circ \phi\right)-\sum_{i}\left(\rho(w) f_{i}\right)\left(v_{i}^{\prime} \circ \phi\right)$.

Obviously, every homomorphism of Lie algebras is a homomorphism of Lie algebroids when the Lie algebras are considered as Lie algebroids, and the tangent map $T \phi: T M \rightarrow T M^{\prime}$ of a smooth map $\phi: M \rightarrow M^{\prime}$ is a homomorphism of Lie algebroids over $\phi$ (see [10]).

The condition (2) does not depend on the choice of the $\Phi$-decompositions of the sections $v$ and $w$ (see [10]) and if $v$ and $w$ are projectable sections, i.e. $\Phi \circ v=v^{\prime} \circ \phi$ and $\Phi \circ w=w^{\prime} \circ \phi$ for some $v^{\prime}, w^{\prime} \in \Gamma\left(A^{\prime}\right)$, then the condition (2) is given simply by $\Phi \circ[v, w]_{A}=\left[v^{\prime}, w^{\prime}\right]_{A^{\prime}} \circ \phi$.

In general not every section is projectable, but there exist homomorphisms $(\Phi, \phi)$ for which all sections are projectable, for example when the bases of the fiber bundles are equal and $\phi=\mathrm{id}_{M}$. In this last case, a homomorphism of Lie algebroids can also be defined in the following way:

Definition 2.2. A vector bundle morphism $\Phi: A \rightarrow A^{\prime}$ over the identity map on $M$ is a $M$-homomorphism of the Lie algebroid $\left(A, \rho,[\cdot, \cdot]_{A}\right)$ in the Lie algebroid $\left(A^{\prime}, \rho^{\prime},[\cdot, \cdot]_{A^{\prime}}\right)$ if $\rho=\rho^{\prime} \circ \Phi$ and $\Phi\left([v, w]_{A}\right)=[\Phi(v), \Phi(w)]_{A^{\prime}}$ for all $v, w \in \Gamma(A)$. If $A=A^{\prime}$ then we say that $\Phi$ is a $M$-endomorphism of Lie algebroids.

The concept of homomorphism of Lie algebroids was defined by Vaintrob [27] in a different but equivalent way by using the notion of homological vector field in a simple graduated manifold $[8,15]$. If $d_{\rho}$ and $d_{\rho^{\prime}}$ are the exterior derivatives of the two Lie algebroids $\left(A, \rho,[\cdot, \cdot]_{A}\right)$ and $\left(A^{\prime}, \rho^{\prime},[\cdot, \cdot]_{A^{\prime}}\right)$, then Vaintrob proved that: A morphism of vector bundles $\Phi: A \rightarrow A^{\prime}$ is a homomorphism of Lie algebroids if and only if $d_{\rho} \circ \Phi^{*}=\Phi^{*} \circ d_{\rho^{\prime}}$, i.e. $\Phi^{*}$ intertwines both differential operators.

\section{Reduction of Lie algebroids}

Definition 3.1. Let $(\Pi, \pi):(A, p, M) \rightarrow(\widehat{A}, \widehat{p}, \widehat{M})$ be a surjective morphism of vector bundles, where $A$ and $\widehat{A}$ are endowed with Lie algebroid structures $\left(\rho,[\cdot, \cdot]_{A}\right)$ and $\left(\widehat{\rho},[\cdot, \cdot]_{\widehat{A}}\right)$ with exterior derivatives $d_{\rho}$ and $d_{\widehat{\rho}}$, respectively. The Lie algebroid $\widehat{A}$ is said to be a reduction of the Lie algebroid $A$ if $\Pi$ is a homomorphism of Lie algebroids, i.e. if $d_{\rho} \circ \Pi^{*}=\Pi^{*} \circ d_{\widehat{\rho}}$. 
Recall that a section of $(\Pi, \pi)$ is a vector bundle morphism $(S, s)$ : $(\widehat{A}, \widehat{p}, \widehat{M}) \rightarrow(A, p, M)$ such that $\Pi \circ S=\operatorname{id}_{\widehat{A}}$ and $\pi \circ s=\operatorname{id}_{\widehat{M}}$. In the following definition we generalize this concept:

Definition 3.2. We call section of the morphism $(\Pi, \pi):(A, p, M) \rightarrow$ $(\widehat{A}, \widehat{p}, \widehat{M})$ to a set of linear maps between pairs of fibres $S=\left\{S_{x}^{x^{\prime}}: \widehat{A}_{x^{\prime}} \rightarrow\right.$ $A_{x} \mid x^{\prime}=\pi(x)$ for all $\left.x \in M\right\}$ such that $\Pi_{x} \circ S_{x}^{x^{\prime}}=\operatorname{id}_{\widehat{A}_{x^{\prime}}}$ for all $x^{\prime} \in \widehat{M}$.

Note that, in general, the set $S$ does not define a morphism of vector bundles.

It was shown in [10] that given a surjective morphism of vector bundles $\Pi: A \rightarrow \widehat{A}$ over $\pi: M \rightarrow \widehat{M}$, all the sections of $A$ are linear combinations of $\Pi$-projectable sections modulo $\Gamma(\operatorname{Ker} \Pi)$, i.e. given $v \in \Gamma(A)$ there exist a finite set of sections $v_{i} \in \Gamma^{\Pi}(A)$ and a section $z \in \Gamma(\operatorname{Ker} \Pi)$ such that $v=\sum_{i} f_{i} v_{i}+z$, with $f_{i} \in C^{\infty}(M)$. Moreover, it was also shown that, if $T \pi \circ \rho=\widehat{\rho} \circ \Pi$ and, furthermore, if $v \sim v^{\prime}$ and $w \sim w^{\prime}$ imply $[v, w]_{A} \sim\left[v^{\prime}, w^{\prime}\right]_{\widehat{A}}$ for all $v, w \in \Gamma^{\Pi}(A)$, then, $\Pi$ is a homomorphism of Lie algebroids.

Proposition 3.3. If $(\Pi, \pi):(A, p, M) \rightarrow(\widehat{A}, \widehat{p}, \widehat{M})$ is a homomorphism of Lie algebroids, then the following conditions are satisfied:

(a) $\rho(\operatorname{Ker} \Pi) \subset \operatorname{Ker} T \pi$;

(b) $\Gamma($ Ker $\Pi)$ is a Lie subalgebra of $\left(\Gamma(A),[\cdot, \cdot]_{A}\right)$.

Proof: Let $(\Pi, \pi)$ be a homomorphism of Lie algebroids. If $v \in \operatorname{Ker} \Pi$, then, by the homomorphism condition $T \pi \circ \rho=\widehat{\rho} \circ \Pi$ we have $T \pi \circ \rho(v)=0$, that is, $v \in \operatorname{Ker}(T \pi \circ \rho)$ and therefore $\rho(\operatorname{Ker} \Pi) \subset \operatorname{Ker}(T \pi)$. Consider $v, w \in$ $\Gamma(\operatorname{Ker} \Pi)$, then the homomorphism condition (2) implies that $\Pi \circ[v, w]_{A}=0$, that is, $[v, w]_{A} \in \Gamma(\operatorname{Ker} \Pi)$.

Let $\left(A, \rho,[\cdot, \cdot]_{A}\right)$ be a Lie algebroid over $M$ and $(\Pi, \pi):(A, p, M) \rightarrow$ $(\widehat{A}, \widehat{p}, \widehat{M})$ a surjective submersion of vector bundles. We can establish conditions in order for the quotient bundle $(\widehat{A}=A / \operatorname{Ker} \Pi, \widehat{p}, \widehat{M})$ to be endowed with a Lie algebroid structure $\left(\widehat{\rho},[\cdot, \cdot]_{\widehat{A}}\right)$ over $\widehat{M}$, in such a way that $\widehat{A}$ is a reduced Lie algebroid.

Let us assume that the following conditions are satisfied:

(C1) There exists a surjective submersion of vector bundles $(\widetilde{\Pi}, \pi)$ : $\left(A^{*}, \tau, M\right) \rightarrow\left((\widehat{A})^{*}, \tau^{\prime}, \widehat{M}\right)$ such that the set

$$
S=\left\{S_{x}^{x^{\prime}}=\left(\widetilde{\Pi}_{x}\right)^{*}: \widehat{A}_{x^{\prime}} \rightarrow A_{x} \mid \pi(x)=x^{\prime}, x \in M\right\}
$$


is a section of $\Pi$, i.e. $\Pi \circ S=\mathrm{id}_{\widehat{A}}$;

(C2) If $\Sigma: \Gamma(\widehat{A}) \rightarrow \Gamma(A)$ is the map between the spaces of sections defined by $\widetilde{\Pi}$ as follows,

$$
\boldsymbol{\Sigma}\left(v^{\prime}\right)(x):=\left(\widetilde{\Pi}_{x}\right)^{*}\left(v_{\pi(x)}^{\prime}\right)=S_{x}^{\pi(x)}\left(v_{\pi(x)}^{\prime}\right)
$$

for all $x \in M$, then, $\operatorname{Im} \boldsymbol{\Sigma}$ is a Lie subalgebra of the Lie algebra $\left(\Gamma(A),[\cdot, \cdot]_{A}\right)$;

(C3) $d_{\rho}$ satisfies the following relation: $d_{\rho} \circ \Pi^{*} \circ \widetilde{\Pi}=\Pi^{*} \circ \widetilde{\Pi} \circ d_{\rho}$.

With these conditions, we can define a Lie bracket on the space of sections of the vector bundle $\widehat{A}$ and a nilpotent derivation $\widehat{d}$ of degree one on the exterior algebra $\Omega^{\bullet}(\widehat{A})$ which endow $\widehat{A}$ with a Lie algebroid structure. The reduction condition (C3) ensures that the Lie algebroid $\widehat{A}$ is a reduced Lie algebroid of $A$, i.e. $d_{\rho} \circ \Pi^{*}=\Pi^{*} \circ \widehat{d}$.

3.1. Reduced Lie algebroid structure. The exterior derivative of the Lie algebroid $A$ can be used to define an operator $\widehat{d}$ in the exterior algebra $\Omega^{\bullet}(\widehat{A})$ of $\widehat{A}$-forms:

$$
\widehat{d}:=\widetilde{\Pi} \circ d_{\rho} \circ \Pi^{*} .
$$

Here $\widetilde{\Pi}$ represents the extension of the morphism $\widetilde{\Pi}: A^{*} \rightarrow(\widehat{A})^{*}$ to the algebra of $A$-forms: given $\alpha \in \Omega^{k}(A)$ and $v_{1}^{\prime}, \ldots, v_{k}^{\prime} \in \Gamma(\widehat{A})$ then, for each $x \in M$,

$$
\begin{aligned}
\widetilde{\Pi}_{x}\left(\alpha_{x}\right)\left(v_{1 \pi(x)}^{\prime}, \ldots, v_{k \pi(x)}^{\prime}\right) & =\alpha_{x}\left(\left(\widetilde{\Pi}_{x}\right)^{*}\left(v_{1 \pi(x)}^{\prime}\right), \ldots,\left(\widetilde{\Pi}_{x}\right)^{*}\left(v_{k \pi(x)}^{\prime}\right)\right) \\
& =\alpha\left(\Sigma\left(v_{1}^{\prime}\right), \ldots, \Sigma\left(v_{k}^{\prime}\right)\right)(x) .
\end{aligned}
$$

We can show that $\widehat{d}$ is a nilpotent (of order two) derivation of degree one of $\Omega^{\bullet}(\widehat{A})$.

Lemma 3.4. $\widehat{d}$ is a derivation of degree one of the exterior algebra $\Omega^{\bullet}(\widehat{A})$, i.e. it satisfies the condition $\widehat{d}\left(\theta^{\prime} \wedge \omega^{\prime}\right)=\widehat{d} \theta^{\prime} \wedge \omega^{\prime}+(-1)^{r} \theta^{\prime} \wedge \widehat{d} \omega^{\prime}$ for all $\theta^{\prime} \in \Omega^{r}(\widehat{A})$ and $\omega^{\prime} \in \Omega^{\bullet}(\widehat{A})$. Furthermore, $\widehat{d}$ is nilpotent of order two, i.e. it satisfies $\widehat{d}^{2}=0$.

Proof: To prove that $\widehat{d}$ is a derivation of degree one it suffices to use definition (3) and the fact that $d_{\rho}$ is a derivation of degree one. The operator $\widehat{d}$ is a derivation of degree one of $\Omega^{\bullet}(\widehat{A})$, so it defines in a unique way a vector bundle map $\widehat{\rho}: \widehat{A} \rightarrow T \widehat{M}$ and a bracket $[\cdot, \cdot]_{\widehat{A}}$ on the space $\Gamma(\widehat{A})$ : 
(i) $\widehat{\rho}\left(v^{\prime}\right) f=\widehat{d} f\left(v^{\prime}\right)$;

(ii) $\eta\left(\left[v_{1}^{\prime}, v_{2}^{\prime}\right]_{\widehat{A}}\right)=\widehat{\rho}\left(v_{1}^{\prime}\right) \eta\left(v_{2}^{\prime}\right)-\widehat{\rho}\left(v_{2}^{\prime}\right) \eta\left(v_{1}^{\prime}\right)-\widehat{d} \eta\left(v_{1}^{\prime}, v_{2}^{\prime}\right)$;

for all $v^{\prime}, v_{1}^{\prime}, v_{2}^{\prime} \in \Gamma(\widehat{A}), f \in C^{\infty}(\widehat{M})$ and $\eta \in \Omega^{1}(\widehat{A})$. We will show later that the bundle map is given by $\widehat{\rho}=\pi_{*} \circ \rho \circ \Sigma$ and the bracket is defined by $[\cdot, \cdot]_{\widehat{A}} \circ \pi=\Pi \circ[\boldsymbol{\Sigma}(\cdot), \boldsymbol{\Sigma}(\cdot)]_{A}$; by conditions (C1) and (C2) we also prove that the bracket satisfy $\Sigma\left([\cdot, \cdot]_{\widehat{A}}\right)=[\boldsymbol{\Sigma}(\cdot), \boldsymbol{\Sigma}(\cdot)]_{A}$. Then the following condition is satisfied

$$
\widehat{d} \circ \widetilde{\Pi}=\widetilde{\Pi} \circ d_{\rho}
$$

even if (C3) is false. In fact, given $\alpha \in \Omega^{r}(A)$ and $v_{1}^{\prime}, \ldots, v_{r+1}^{\prime} \in \Gamma(\widehat{A})$ we have

$$
\begin{aligned}
\widehat{d} \circ \widetilde{\Pi}(\alpha)\left(v_{1}^{\prime}, \ldots, v_{r+1}^{\prime}\right) \circ \pi= & \sum_{i}(-1)^{i+1}\left[\widehat{\rho}\left(v_{i}^{\prime}\right) \circ \pi\right] \widetilde{\Pi}(\alpha)\left(\ldots, \widehat{v_{i}}, \ldots\right) \\
& +\sum_{i<j}(-1)^{i+j} \widetilde{\Pi}(\alpha)\left(\left[v_{i}^{\prime}, v_{j}^{\prime}\right]_{\widehat{A}}, \ldots, \widehat{v_{i}^{\prime}}, \ldots \widehat{v_{j}^{\prime}}, \ldots\right) \circ \pi \\
= & \sum_{i}(-1)^{i+1}\left[\pi_{*} \circ \rho \circ \boldsymbol{\Sigma}\left(v_{i}^{\prime}\right)\right] \widetilde{\Pi}(\alpha)\left(\ldots, \widehat{v_{i}}, \ldots\right) \\
& +\sum_{i<j}(-1)^{i+j} \alpha\left(\boldsymbol{\Sigma}\left(\left[v_{i}^{\prime}, v_{j}^{\prime}\right]_{\widehat{A}}\right), \ldots, \widehat{\boldsymbol{\Sigma}\left(v_{i}^{\prime}\right)}, \ldots \widehat{\boldsymbol{\Sigma}\left(v_{j}^{\prime}\right)}, \ldots\right) \\
= & \sum_{i}(-1)^{i+1}\left[\rho \circ \boldsymbol{\Sigma}\left(v_{i}^{\prime}\right)\right]\left[\alpha\left(\ldots, \widehat{\boldsymbol{\Sigma}\left(v_{i}\right)}, \ldots\right)\right] \\
& +\sum_{i<j}(-1)^{i+j} \alpha\left(\left[\boldsymbol{\Sigma}\left(v_{i}^{\prime}\right), \boldsymbol{\Sigma}\left(v_{j}^{\prime}\right)\right]_{A}, \ldots, \widehat{\boldsymbol{\Sigma}\left(v_{i}^{\prime}\right)}, \ldots \widehat{\boldsymbol{\Sigma}\left(v_{j}^{\prime}\right)}, \ldots\right) \\
= & d_{\rho} \alpha\left(\boldsymbol{\Sigma}\left(v_{1}^{\prime}\right), \ldots, \boldsymbol{\Sigma}\left(v_{r+1}^{\prime}\right)\right)=\widetilde{\Pi} \circ d_{\rho} \alpha\left(v_{1}^{\prime}, \ldots, v_{r+1}^{\prime}\right) \circ \pi ;
\end{aligned}
$$

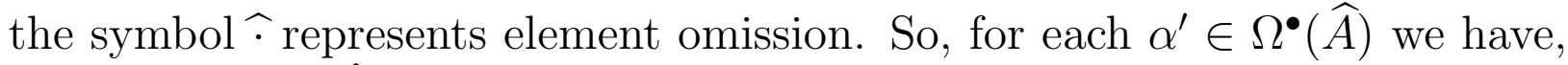
by definition of $\widehat{d}$, that

$$
\widehat{d}\left(\widehat{d} \alpha^{\prime}\right)=\widehat{d}\left(\widetilde{\Pi} \circ d_{\rho} \circ \Pi^{*}\left(\alpha^{\prime}\right)\right) .
$$

Using $\widehat{d} \circ \widetilde{\Pi}=\widetilde{\Pi} \circ d_{\rho}$ and that $d_{\rho}$ is nilpotent, we obtain $\widehat{d}^{2}=0$.

The previous result can also be stated as follows:

Theorem 3.5. The operator $\widehat{d}$ is a homological vector field of degree one of the graded manifold $\widehat{\mathcal{M}}=\left(\widehat{M}, \Omega^{\bullet}(\widehat{A})\right)$. 
According to Vaintrob [27], the homological vector field $\widehat{d}$ defines a Lie algebroid structure in the vector bundle $\widehat{A}$, for which $\widehat{d}$ is the exterior derivative of the Lie algebroid; the anchor is the above defined bundle map $\widehat{\rho}$ and the Lie bracket on sections is given by $[\cdot, \cdot]_{\widehat{A}}$. From the relation (4) and the reduction condition (C3) we can conclude that

$$
\Pi^{*} \circ \widehat{d}=d_{\rho} \circ \Pi^{*} \text {. }
$$

Therefore, $\widehat{A}$ is a reduced Lie algebroid of $A$. A Lie algebroid $A$ satisfying the above conditions of reduction, is called reducible Lie algebroid.

Proposition 3.6. The anchor of the Lie algebroid $\widehat{A}$ is given by $\widehat{\rho}=T \pi \circ \rho \circ S$.

Proof: Let $f^{\prime} \in C^{\infty}(\widehat{M})$ and $v^{\prime} \in \Gamma(\widehat{A})$. For all $x \in M$, we have

$$
\begin{aligned}
\widehat{d} f^{\prime}\left(v^{\prime}\right) \circ \pi(x) & :=\widetilde{\Pi}_{x}\left(d_{\rho}\left(f^{\prime} \circ \pi\right)(x)\right)\left(v_{\pi(x)}^{\prime}\right) \\
& =d_{\rho}\left(f^{\prime} \circ \pi\right)\left(\boldsymbol{\Sigma}\left(v^{\prime}\right)\right)(x) \\
& =d f^{\prime}(\pi(x))\left(T \pi \circ \rho \circ \boldsymbol{\Sigma}\left(v^{\prime}\right)(x)\right) .
\end{aligned}
$$

On the other hand,

$$
\widehat{d} f^{\prime}\left(v^{\prime}\right) \circ \pi(x)=d f^{\prime}\left(\widehat{\rho}\left(v^{\prime}\right)\right) \circ \pi(x),
$$

and, since $f^{\prime}$ is arbitrary, we can conclude that

$$
\widehat{\rho}\left(v^{\prime}\right) \circ \pi(x)=T \pi \circ \rho \circ \boldsymbol{\Sigma}\left(v^{\prime}\right)(x)
$$

for all $x \in M$, that is, $\rho \circ \boldsymbol{\Sigma}\left(v^{\prime}\right)$ is a vector field $T \pi$-projectable onto $\widehat{\rho}\left(v^{\prime}\right)$. Then, for every $v^{\prime} \in \Gamma(\widehat{A})$ and $x \in M$, we have

$\widehat{\rho}\left(v^{\prime}\right) \circ \pi(x)=T \pi \circ \rho\left(\Sigma\left(v^{\prime}\right)(x)\right)=T_{x} \pi \circ \rho_{x} \circ\left(\widetilde{\Pi}_{x}\right)^{*}\left(v_{\pi(x)}^{\prime}\right)=T_{x} \pi \circ \rho_{x} \circ S_{x}^{\pi(x)}\left(v_{\pi(x)}^{\prime}\right)$ that is,

$$
\widehat{\rho}_{\pi(x)}=T_{x} \pi \circ \rho_{x} \circ S_{x}^{\pi(x)} .
$$

Therefore, the anchor is given by $\widehat{\rho}=T \pi \circ \rho \circ S$.

From now on the operator $\widehat{d}$ will be denoted by $d_{\widehat{\rho}}$. We can check that $\widehat{\rho}$ is a morphism of vector bundles over the identity map on $\widehat{M}$,

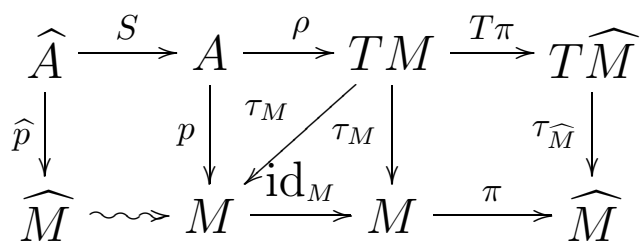


which furnishes a morphism between the spaces of sections, that we represent by the same symbol: $\widehat{\rho}=\pi_{*} \circ \rho \circ \Sigma$, where $\pi_{*}: \Gamma^{T \pi}(T M) \subset \mathfrak{X}(M) \rightarrow \mathfrak{X}(\widehat{M})$ is a morphism applying each $T \pi$-projectable vector field $X \in \mathfrak{X}(M)$ in the $T \pi$-related vector field $X^{\prime} \in \mathfrak{X}(\widehat{M})$, i.e. if $T \pi \circ X=X^{\prime} \circ \pi$ then $\pi_{*} X=X^{\prime}$.

Proposition 3.7. The Lie bracket on the space of sections of the vector bundle $\widehat{A}$ is given by $[\cdot, \cdot]_{\widehat{A}} \circ \pi:=\Pi \circ[\boldsymbol{\Sigma}(\cdot), \boldsymbol{\Sigma}(\cdot)]_{A}$ and satisfy the condition:

$$
\Sigma\left(\left[v^{\prime}, w^{\prime}\right]_{\widehat{A}}\right)=\left[\Sigma\left(v^{\prime}\right), \Sigma\left(w^{\prime}\right)\right]_{A}
$$

for all $v^{\prime}, w^{\prime} \in \Gamma(\widehat{A})$.

Proof: By the above proposition and by (ii) given in lemma 3.4, we show that the Lie bracket is given by $[\cdot, \cdot]_{\widehat{A}} \circ \pi:=\Pi \circ[\Sigma(\cdot), \Sigma(\cdot)]_{A}$. For $v^{\prime}, w^{\prime} \in \Gamma(\widehat{A})$ and $x \in M$, we have

$$
\boldsymbol{\Sigma}\left(\left[v^{\prime}, w^{\prime}\right]_{\widehat{A}}\right)(x)=S_{x}^{\pi(x)}\left(\left[v^{\prime}, w^{\prime}\right]_{\widehat{A}}(\pi(x))\right)=S_{x}^{\pi(x)}\left(\Pi \circ\left[\boldsymbol{\Sigma}\left(v^{\prime}\right), \boldsymbol{\Sigma}\left(w^{\prime}\right)\right]_{A}(x)\right) ;
$$

by (C2) there exists $u^{\prime} \in \Gamma(\widehat{A})$ such that $\left[\boldsymbol{\Sigma}\left(v^{\prime}\right), \boldsymbol{\Sigma}\left(w^{\prime}\right)\right]_{A}(x)=\boldsymbol{\Sigma}\left(u^{\prime}\right)(x)$. So by $(\mathrm{C} 1)$ we have

$$
\boldsymbol{\Sigma}\left(\left[v^{\prime}, w^{\prime}\right]_{\widehat{A}}\right)(x)=S_{x}^{\pi(x)}\left(u^{\prime}(\pi(x))\right)=\boldsymbol{\Sigma}\left(u^{\prime}\right)(x)=\left[\boldsymbol{\Sigma}\left(v^{\prime}\right), \boldsymbol{\Sigma}\left(w^{\prime}\right)\right]_{A}(x) .
$$

3.2. Third reduction condition of a Lie algebroid. In this section, we will show that, if the reduction conditions (C1) and (C2) are satisfied, the reduction condition $(\mathrm{C} 3)$ is equivalent to the three following conditions:

(1) $\rho(\operatorname{Ker} \Pi) \subset \operatorname{Ker} T \pi$;

(2) $[\operatorname{Im} \Sigma, \Gamma(\operatorname{Ker} \Pi)]_{A} \subset \Gamma(\operatorname{Ker} \Pi)$;

(3) $\Gamma(\operatorname{Ker} \Pi)$ is a Lie subalgebra of $\left(\Gamma(A),[\cdot, \cdot]_{A}\right)$.

Proposition 3.8. Let us suppose that $(\Pi, \pi):(A, p, M) \rightarrow(\widehat{A}, \widehat{p}, \widehat{M})$ is a homomorphism of Lie algebroids. Then, if (C1) holds the conditions (1), (2) and (3) are satisfied.

Proof: We know, from proposition 3.3, that if $(\Pi, \pi)$ is a homomorphism of Lie algebroids, the conditions (1) and (3) are satisfied and so we only need to check condition (2). For every section $v^{\prime} \in \Gamma(\widehat{A})$ and $x \in M$, we have

$$
\Pi \circ \Sigma\left(v^{\prime}\right)(x)=\Pi_{x} \circ S_{x}^{\pi(x)}\left(v_{\pi(x)}^{\prime}\right),
$$

and by condition $(\mathrm{C} 1)$, we also have

$$
\Pi \circ \Sigma\left(v^{\prime}\right)(x)=v_{\pi(x)}^{\prime}=v^{\prime} \circ \pi(x) .
$$


Thus, all sections in the image of $\boldsymbol{\Sigma}$ are $\Pi$-projectable. For $w \in \Gamma(\operatorname{Ker} \Pi)$, condition (2) implies that $\Pi \circ\left[\boldsymbol{\Sigma}\left(v^{\prime}\right), w\right]_{A}=0$.

Using the fact that every section $v$ of $A$ can be written in the form $v=$ $\sum_{i} f_{i} \boldsymbol{\Sigma}\left(v_{i}^{\prime}\right)+z$, with $z \in \Gamma(\operatorname{Ker} \Pi), f_{i} \in C^{\infty}(M)$ and $v_{i}^{\prime} \in \Gamma(\widehat{A})$, we can prove the following result:

Proposition 3.9. Let us suppose that (C1), (C2) and the conditions (1), (2) and (3) hold. Then, $(\Pi, \pi)$ is a homomorphism of Lie algebroids.

Proof: Let $v=\sum_{i} f_{i} \boldsymbol{\Sigma}\left(v_{i}^{\prime}\right)+z$ and $w=\sum_{j} g_{j} \boldsymbol{\Sigma}\left(w_{j}^{\prime}\right)+y$ be sections of $A$ with $f_{i}, g_{j} \in C^{\infty}(M), v_{i}^{\prime}, w_{j}^{\prime} \in \Gamma(\widehat{A})$ and $z, y \in \Gamma(\operatorname{Ker} \Pi)$. Thus,

$$
[v, w]_{A}=\sum_{i, j \in I}\left[f_{i} \boldsymbol{\Sigma}\left(v_{i}^{\prime}\right), g_{j} \boldsymbol{\Sigma}\left(w_{j}^{\prime}\right)\right]_{A}+\sum_{i}\left[f_{i} \boldsymbol{\Sigma}\left(v_{i}^{\prime}\right), y\right]_{A}+\sum_{j}\left[z, g_{j} \boldsymbol{\Sigma}\left(w_{j}^{\prime}\right)\right]_{A}+[z, y]_{A} \text {. }
$$

The Leibniz property for the Lie bracket $[\cdot, \cdot]_{A}$ implies that

$$
\begin{aligned}
{[v, w]_{A}=} & \sum_{i, j} f_{i} g_{j}\left[\boldsymbol{\Sigma}\left(v_{i}^{\prime}\right), \boldsymbol{\Sigma}\left(w_{j}^{\prime}\right)\right]_{A}+\rho\left(\sum_{i} f_{i} \boldsymbol{\Sigma}\left(v_{i}^{\prime}\right)\right) g_{j} \boldsymbol{\Sigma}\left(w_{j}^{\prime}\right) \\
& -\rho\left(\sum_{j} g_{j} \boldsymbol{\Sigma}\left(w_{j}^{\prime}\right)\right) f_{i} \boldsymbol{\Sigma}\left(v_{i}^{\prime}\right)+\sum_{i} f_{i}\left[\boldsymbol{\Sigma}\left(v_{i}^{\prime}\right), y\right]_{A}-\sum_{i} \rho(y) f_{i} \boldsymbol{\Sigma}\left(v_{i}^{\prime}\right) \\
& +\sum_{j} g_{j}\left[z, \boldsymbol{\Sigma}\left(w_{j}^{\prime}\right)\right]_{A}+\sum_{j} \rho(z) g_{j} \boldsymbol{\Sigma}\left(w_{j}^{\prime}\right)+[z, y]_{A}
\end{aligned}
$$

and one can obtain, from the conditions (2) and (3),

$$
\begin{aligned}
\Pi \circ[v, w]_{A}= & \sum_{i, j} f_{i} g_{j} \Pi \circ\left[\boldsymbol{\Sigma}\left(v_{i}^{\prime}\right), \boldsymbol{\Sigma}\left(w_{j}^{\prime}\right)\right]_{A}+\sum_{j} \rho(v) g_{j}\left(\Pi \circ \boldsymbol{\Sigma}\left(w_{j}^{\prime}\right)\right) \\
& -\sum_{i} \rho(w) f_{i}\left(\Pi \circ \boldsymbol{\Sigma}\left(v_{i}^{\prime}\right)\right) \\
= & \sum_{i, j} f_{i} g_{j}\left(\left[v_{i}^{\prime}, w_{j}^{\prime}\right]_{\widehat{A}} \circ \pi\right)+\sum_{j} \rho(v) g_{j}\left(w_{j}^{\prime} \circ \pi\right)-\sum_{i} \rho(w) f_{i}\left(v_{i}^{\prime} \circ \pi\right) .
\end{aligned}
$$

Now, we have to check that $T \pi \circ \rho=\widehat{\rho} \circ \Pi$. As a consequence of condition (1), we only need to prove the equality for sections of $A$ of the form $v=\Sigma\left(v^{\prime}\right)$ for $v^{\prime} \in \Gamma(A)$. Because of the reduction condition (C1) we have

$$
\widehat{\rho} \circ \Pi \circ \boldsymbol{\Sigma}\left(v^{\prime}\right)(x)=\widehat{\rho} \circ \Pi_{x} \circ S_{x}^{\pi(x)}\left(v^{\prime}(\pi(x))=\widehat{\rho}\left(v^{\prime}\right)(\pi(x)),\right.
$$

and $\widehat{\rho}\left(v^{\prime}\right)(\pi(x)):=T \pi \circ \rho \circ \Sigma\left(v^{\prime}\right)(x)$ for all $x \in M$; therefore, $\widehat{\rho} \circ \Pi \circ \Sigma\left(v^{\prime}\right)(x)=$ $T \pi \circ \rho \circ \Sigma\left(v^{\prime}\right)(x)$. 
Assuming that the conditions $(\mathrm{C} 1)$ and $(\mathrm{C} 2)$ hold, it is easy to prove that condition (C3) is satisfied if and only if $\Pi$ is a homomorphism of Lie algebroids. Therefore, condition (C3) is equivalent to the conditions (1), (2) and (3).

Next, we prove that the anchor and the bracket do not depend on the choice of the map $\widetilde{\Pi}$.

Proposition 3.10. The reduced Lie algebroid structure $\left(\widehat{\rho},[\cdot, \cdot]_{\widehat{A}}\right)$ in $\widehat{A}$ is independent on the choice of the morphism in the reduction conditions of the Lie algebroid $\left(A, \rho,[\cdot, \cdot]_{A}\right)$.

Proof: Let $\bar{\Pi}$ be a different morphism satisfying the reduction conditions. In order to prove the uniqueness of the reduced anchor, we have to show that

$$
T \pi \circ \rho \circ \widetilde{S}\left(v^{\prime}\right)=T \pi \circ \rho \circ \bar{S}\left(v^{\prime}\right)
$$

for all $v^{\prime} \in \widehat{A}$, where $\widetilde{S}$ and $\bar{S}$ are sections of $\Pi$ defined, respectively, by $\widetilde{\Pi}$ and $\bar{\Pi}$. Note that $\widetilde{S}\left(v^{\prime}\right)-\bar{S}\left(v^{\prime}\right) \in \operatorname{Ker} \Pi$, and since $\operatorname{Ker} \Pi \subset \operatorname{Ker}(T \pi \circ \rho)$, (6) holds. As far as the bracket is concerned we have to show that

$$
\Pi \circ\left[\boldsymbol{\Sigma}\left(v^{\prime}\right), \boldsymbol{\Sigma}\left(w^{\prime}\right)\right]_{A}=\Pi \circ\left[\overline{\boldsymbol{\Sigma}}\left(v^{\prime}\right), \overline{\boldsymbol{\Sigma}}\left(w^{\prime}\right)\right]_{A}
$$

for all $v^{\prime}, w^{\prime} \in \Gamma(\widehat{A})$. Since $\Sigma\left(v^{\prime}\right)-\bar{\Sigma}\left(v^{\prime}\right) \in \Gamma(\operatorname{Ker} \Pi)$ for all $v^{\prime} \in \Gamma(\widehat{A})$, there exists $z \in \Gamma($ Ker $\Pi)$ such that $\boldsymbol{\Sigma}\left(v^{\prime}\right)=\bar{\Sigma}\left(v^{\prime}\right)+z$, and then

$$
\begin{aligned}
\Pi \circ\left[\boldsymbol{\Sigma}\left(v^{\prime}\right), \boldsymbol{\Sigma}\left(w^{\prime}\right)\right]_{A}= & \Pi \circ\left[\overline{\boldsymbol{\Sigma}}\left(v^{\prime}\right)+z_{1}, \overline{\boldsymbol{\Sigma}}\left(w^{\prime}\right)+z_{2}\right]_{A} \\
= & \Pi \circ\left(\left[\overline{\boldsymbol{\Sigma}}\left(v^{\prime}\right), \overline{\boldsymbol{\Sigma}}\left(w^{\prime}\right)\right]_{A}+\left[\overline{\boldsymbol{\Sigma}}\left(v^{\prime}\right), z_{2}\right]_{A}\right) \\
& +\Pi \circ\left(\left[z_{1}, \overline{\boldsymbol{\Sigma}}\left(w^{\prime}\right)\right]_{A}+\left[z_{1}, z_{2}\right]_{A}\right) .
\end{aligned}
$$

Finally, since $[\operatorname{Im} \Sigma, \Gamma(\operatorname{Ker} \Pi)]_{A} \subset \Gamma(\operatorname{Ker} \Pi)$ and $\Gamma(\operatorname{Ker} \Pi)$ is a Lie subalgebra of $\Gamma(A)$, we have

$$
\Pi \circ\left[\boldsymbol{\Sigma}\left(v^{\prime}\right), \boldsymbol{\Sigma}\left(w^{\prime}\right)\right]_{A}=\Pi \circ\left[\overline{\boldsymbol{\Sigma}}\left(v^{\prime}\right), \overline{\boldsymbol{\Sigma}}\left(w^{\prime}\right)\right]_{A} .
$$

3.3. The reduction theorem. We can summarize almost all the information about Lie algebroid reduction in a unique result.

Theorem 3.11. Let $\left(A, \rho,[\cdot, \cdot]_{A}\right)$ be a Lie algebroid over $M, d_{\rho}$ its exterior derivative and $(\Pi, \pi):(A, p, M) \rightarrow(\widehat{A}, \widehat{p}, \widehat{M})$ a surjective submersion of vector bundles, satisfying the following conditions: 
(C1) There exists a surjective submersion of vector bundles $(\widetilde{\Pi}, \pi)$ : $\left(A^{*}, \tau, M\right) \rightarrow\left((\widehat{A})^{*}, \widehat{\tau}, \widehat{M}\right)$ for which the set

$$
S=\left\{S_{x}^{x^{\prime}}=\left(\widetilde{\Pi}_{x}\right)^{*}: \widehat{A}_{x^{\prime}} \rightarrow A_{x} \mid \pi(x)=x^{\prime}, x \in M\right\}
$$

is a section of $\Pi$;

(C2) If $\Sigma: \Gamma(\widehat{A}) \rightarrow \Gamma(A)$ is the map between sections induced by $\widetilde{\Pi}$, then, $\operatorname{Im} \Sigma$ is a Lie subalgebra of $\Gamma(A)$.

Then, the quotient vector bundle $(\widehat{A}, \widehat{p}, \widehat{M})$ is a Lie algebroid whose exterior derivative $d_{\widehat{\rho}}$ satisfies the condition $d_{\widehat{\rho}} \circ \widetilde{\Pi}=\widetilde{\Pi} \circ d_{\rho}$ and whose structure $\left(\widehat{\rho},[\cdot, \cdot]_{\widehat{A}}\right)$ is given by:

(i) $\widehat{\rho}=T \pi \circ \rho \circ S$;

(ii) $[\cdot, \cdot]_{\widehat{A}} \circ \pi=\Pi \circ[\Sigma(\cdot), \Sigma(\cdot)]_{A}$.

Moreover, the triple $(\widehat{A}, \widehat{p}, \widehat{M})$ is a reduced Lie algebroid of $A$ when one of the following equivalent conditions holds:

(a) $d_{\rho} \circ \Pi^{*} \circ \widetilde{\Pi}=\Pi^{*} \circ \widetilde{\Pi} \circ d_{\rho}$;

(b) $\Pi$ is a homomorphism of Lie algebroids;

(c) the following conditions are satisfied:

(1) $\rho(\operatorname{Ker} \Pi) \subset \operatorname{Ker} T \pi$;

(2) $[\operatorname{Im} \Sigma, \Gamma(\operatorname{Ker} \Pi)]_{A} \subset \Gamma(\operatorname{Ker} \Pi)$;

(3) $\Gamma($ Ker $\Pi)$ is a Lie subalgebra of $\left(\Gamma(A),[\cdot, \cdot]_{A}\right)$.

\subsection{Some examples of reduction.}

3.4.1. Poisson manifold. Let $(M, \Lambda)$ be a Poisson manifold, $\left(T^{*} M, \Lambda^{\sharp}\right.$, $\left.[\cdot, \cdot]_{T^{*} M}\right)$ the associated Lie algebroid and $(\Pi, \pi):\left(T^{*} M, \tau, M\right) \rightarrow(\widehat{A}, \widehat{\tau}, \widehat{M})$ a surjective submersion, that defines the quotient vector bundle $\widehat{A}=$ $T^{*} M / \operatorname{Ker} \Pi$. Suppose that $A=T^{*} M$ satisfies the conditions of the reduction theorem. Then, we can define a Lie algebroid structure in $\widehat{A}$ by means of:

(i) $\widehat{\rho}=T \pi \circ \Lambda^{\sharp} \circ S$;

(ii) $[\cdot, \cdot]_{\widehat{A}} \circ \pi=\Pi \circ[\Sigma(\cdot), \Sigma(\cdot)]_{T^{*} M}$.

Proposition 3.12. Let us suppose that the reduction conditions hold for $\widetilde{\Pi}=T \pi$. Let $f, g$ be smooth functions in $M$ such that their differentials $d f$ and $d g$ vanish in $C_{M}=\operatorname{Ker} T \pi$. Then, $d\{f, g\}_{M}$ vanishes in $C_{M}$. 
Proof: Let us denote by $C_{M}^{0}$ the annihilator of $C_{M}$. If $d f \in C_{M}^{0}$ then there exists a smooth function $f^{\prime}$ in $\widehat{M}$, such that, $f=f^{\prime} \circ \pi$. Thus, $d f(x)=$ $\left(\pi^{*}\right)_{\pi(x)}\left(d f^{\prime}(\pi(x))\right)=\left(\widetilde{\Pi}_{x}\right)^{*}\left(d f^{\prime}(\pi(x))\right)=\boldsymbol{\Sigma}\left(d f^{\prime}\right)(x)$. In the same way, given $d g \in C_{M}^{0}$ there is a smooth function $g^{\prime}$ in $\widehat{M}$, such that, $d g=\Sigma\left(d g^{\prime}\right)$. Using the condition $(\mathrm{C} 2)$, we show that

$$
\begin{aligned}
\chi\left(d\{f, g\}_{M}\right) & =\chi\left(\left[\boldsymbol{\Sigma}\left(d f^{\prime}\right), \boldsymbol{\Sigma}\left(d g^{\prime}\right)\right]_{T^{*} M}\right) \\
& =\chi\left(\boldsymbol{\Sigma}\left(\left[d f^{\prime}, d g^{\prime}\right]_{T^{*} \widehat{M}}\right)\right) \\
& =\widehat{\chi}\left(\left[d f^{\prime}, d g^{\prime}\right]_{T^{*} \widehat{M}}\right) \circ \widetilde{\Pi} .
\end{aligned}
$$

where $\chi: \Gamma\left(T^{*} M\right) \rightarrow L(T M)$ and $\widehat{\chi}: \Gamma\left(T^{*} \widehat{M}\right) \rightarrow L(T \widehat{M})$ with $L(T M)$ and $L(T \widehat{M})$ being the spaces of linear functions on $T M$ and $T \widehat{M}$, respectively. As the morphism $\widetilde{\Pi}=T \pi$ is zero in $C_{M}$, then $d\{f, g\}_{M} \in C_{M}^{0}$.

Thus, applying the reduction of Marsden-Ratiu ([20]) to the Poisson manifold $M$, where the completely integrable distribution is given by $C_{M}=$ $\operatorname{Ker} T \pi$, we can define in $\widehat{M}$ (the manifold of leaves) a Poisson bracket. A Poisson structure in $\widehat{M}$ defines a Lie algebroid structure in $T^{*} \widehat{M}$ that coincides with the structure defined by the conditions (i) e (ii).

3.4.2. Group action. Let $\phi: M \times G \rightarrow M$ be a right-action of the Lie group $G$ on the manifold $M$ and consider the Lie algebroid structure in the trivial fiber bundle $M \times \mathfrak{g} \rightarrow M$ associated to such action $\phi$, where $\mathfrak{g}$ is the Lie algebra of $G$, as given in the example 2.2. Let $(\Pi, \pi):(M \times \mathfrak{g}, \tau, M) \rightarrow(N \times \mathfrak{g}, \widehat{\tau}, N)$ be a surjective submersion of vector bundles defined by $\Pi=\left(\pi, \operatorname{id}_{\mathfrak{g}}\right)$ and $(\widetilde{\Pi}, \pi):\left(M \times \mathfrak{g}^{*}, \tau, M\right) \rightarrow\left(N \times \mathfrak{g}^{*}, \widehat{\tau}, N\right)$ a surjective submersion of vector bundles given by $\widetilde{\Pi}=\left(\pi, \mathrm{id}_{\mathfrak{g}^{*}}\right)$. Suppose also that $\operatorname{Im} \Sigma$ is a subalgebra of the space of sections of $M \times \mathfrak{g}$. Since the reduction conditions (C1), (C2) and (C3) hold, $N \times \mathfrak{g}$ is endowed with a Lie algebroid structure whose anchor and Lie bracket are given by:

(i) $\widehat{\rho}=T \pi \circ \rho \circ S$;

(ii) $[\cdot, \cdot]_{N \times \mathfrak{g}} \circ \pi=\Pi \circ[\Sigma(\cdot), \Sigma(\cdot)]_{M \times \mathfrak{g}} \cdot$

Note that, $S_{x}^{\pi(x)}\left(X_{\pi(x)}^{\prime}\right)=S_{x}^{\pi(x)}\left(\pi(x), \overline{X^{\prime}}(\pi(x))\right)=\left(x, \overline{X^{\prime}}(\pi(x))\right)$ for all $x \in$ $M$. Thus, the anchor is given by $\widehat{\rho}\left(X^{\prime}\right)(\pi(x))=T_{x} \pi\left(X_{M}(x)\right)$, with $X=$ $\overline{X^{\prime}}(\pi(x)) \in \mathfrak{g}$, and the bracket is given by

$$
\left[X^{\prime}, Y^{\prime}\right]_{N \times \mathfrak{g}}\left(x^{\prime}\right)=\left(x^{\prime},\left[\overline{X^{\prime}}\left(x^{\prime}\right), \overline{Y^{\prime}}\left(x^{\prime}\right)\right]_{\mathfrak{g}}+\widehat{\rho}\left(X^{\prime}\right) \overline{Y^{\prime}}\left(x^{\prime}\right)-\widehat{\rho}\left(Y^{\prime}\right) \overline{X^{\prime}}\left(x^{\prime}\right)\right)
$$


for all $x^{\prime} \in N$ and all $X^{\prime}, Y^{\prime} \in \Gamma(N \times \mathfrak{g})$. In the above conditions, only the homomorphism $\widetilde{\Pi}=\left(\pi, \mathrm{id}_{\mathfrak{g}^{*}}\right)$ satisfies the reduction conditions of the Lie algebroid $M \times \mathfrak{g} \rightarrow M$.

3.4.3. Reduction of degenerated Lagrangian systems. Let us consider a Lagrangian $L \in C^{\infty}(T Q)$ satisfying the following conditions (see [2]):

(A1) the Cartan 2-form $\omega_{L}$ is presymplectic, i.e. it is a constant rank closed form;

(A2) the Lagrangian $L$ admits a global dynamics, i.e. there exists a globally defined vector field $X$ on $T Q$ satisfying the dynamical equation $i(X) \omega_{L}=d E_{L}$

(A3) the foliation defined by $\omega_{L}$ is regular, i.e. the quotient space has a differentiable manifold structure and the projection $\Pi: T Q \rightarrow$ $T Q / \operatorname{Ker} \omega_{L}$ is a surjective submersion.

The distribution $D$ of $Q$ given by $D=\left\{X \in \mathfrak{X}(Q) \mid X^{c} \in \operatorname{Ker} \omega_{L}\right\}$ is integrable because it is involutive and has constant rank (theorem of Frobenius). Let us suppose also that

(A4) the distribution $D$ defines a regular foliation of $Q$, i.e. the space of the leaves $\widehat{Q}=Q / D$ admits a structure of manifold whose projection $\pi: Q \rightarrow \widehat{Q}$ is a surjective submersion.

Under these conditions, we can prove that there exists a unique vector bundle structure in the quotient manifold $\widehat{T Q}=T Q / \operatorname{Ker} \omega_{L}$ such that the following diagram commute:

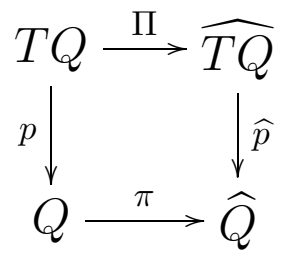

The projection $\widehat{p}: \widehat{T Q} \rightarrow \widehat{Q}$ is given by $\widehat{p}([X])=\pi(p(X))$ for each $X \in T Q$ such that $\Pi(X)=[X]$, where $p: T Q \rightarrow Q$ is the canonical projection of the tangent bundle $T Q$ onto $Q$. The set $\widehat{T Q}_{\widehat{q}}=\widehat{p}^{-1}(\widehat{q})$ is endowed with vector bundle structure for all $\widehat{q} \in \widehat{Q}$.

We know that the tangent bundle $T Q$ is a Lie algebroid over $Q$ whose anchor is given by the identity on $T Q$ and the bracket on sections $[\cdot, \cdot]$ is the usual bracket of vector fields on $Q$. If the surjective submersion of vector bundles $(\Pi, \pi):(T Q, p, Q) \rightarrow(\widehat{T Q}, \widehat{p}, \widehat{Q})$ satisfies the Lie algebroid reduction 
conditions then the bundle $\widehat{T Q}$ is endowed with a Lie algebroid structure given by:

(i) $\widehat{\rho}=T \pi \circ S$;

(ii) $[\cdot, \cdot]_{\widehat{T Q}} \circ \pi=\Pi \circ[\Sigma(\cdot), \Sigma(\cdot)]$.

For example, when $\Pi=T \pi$ we have that the tangent bundle $\widehat{T Q}=T \widehat{Q}$ is endowed with the usual Lie algebroid structure and in this case it is a reduced Lie algebroid of $T Q$.

\section{Reduction of Lie co-algebroids}

It is possible to show that the reduction of a Lie algebroid implies the reduction of the corresponding Lie co-algebroid according to Marsden-Ratiu reduction procedure [20]. However, as it will be shown in this section, the converse is not true in general.

Let $\left(A, \rho,[\cdot, \cdot]_{A}\right)$ be a Lie algebroid over $M$ and $\left(A^{*}, \Lambda_{A^{*}}\right)$ the associated Lie co-algebroid. Let us consider a subbundle $C$ of $T A^{*}$ satisfying the following conditions:

1. $C$ is a completely integrable distribution of $A^{*}$, such that, the set $\widehat{A^{*}}$ of the leaves of the foliation defined in $A^{*}$ by $C$ is a differentiable manifold, and the canonical projection $(\widetilde{\Pi}, \pi):\left(A^{*}, \tau, M\right) \rightarrow\left(\widehat{A^{*}}, \widehat{\tau}, \widehat{M}\right)$ is a surjective submersion of vector bundles;

2. for every pair of affine functions, $F, G \in C^{\infty}\left(A^{*}\right)$ whose differentials belong to the annihilator $C^{0}$ of $C$, the differential of the bracket of these functions $d\{F, G\}_{A^{*}}$ still belongs to $C^{0}$.

According to Marsden-Ratiu reduction theory developed in [20], the bundle $\left(\widehat{A^{*}}, \widehat{\tau}, \widehat{M}\right)$ is endowed with a Poisson structure, $\{\cdot, \cdot\}_{\wedge}$, given by:

$$
\{\widehat{F} \circ \widetilde{\Pi}, \widehat{G} \circ \widetilde{\Pi}\}_{A^{*}}=\{\widehat{F}, \widehat{G}\}_{\wedge} \circ \widetilde{\Pi},
$$

for all $\widehat{F}, \widehat{G} \in C^{\infty}\left(\widehat{A^{*}}\right)$. The pair $\left(A^{*}, C\right)$ is called reducible Poisson manifold. Note that, in the given hypotheses, the condition $\Lambda^{\sharp}\left(C^{0}\right) \subset T A^{*}+C \subset T^{*} A$ holds and therefore the reduced bracket $\{\cdot, \cdot\}_{\wedge}$ does exist.

Let $L\left(\widehat{A^{*}}\right)$ and $L\left(A^{*}\right)$ denote the spaces of linear functions on $\widehat{A^{*}}$ and $A^{*}$, respectively. Given a linear function $F=\chi(v)$ on $A^{*}$ with $v \in \Gamma(A)$ such that $d F \in C^{0}$, there exists a linear function $\widehat{F}=\widehat{\chi}\left(v^{\prime}\right)$ defined on $\widehat{A^{*}}$, with $v^{\prime} \in$ $\Gamma\left(\left(\widehat{A^{*}}\right)^{*}\right)$, such that $F=\widehat{F} \circ \widetilde{\Pi}=\widehat{\chi}\left(v^{\prime}\right) \circ \widetilde{\Pi}=\chi\left(\Sigma\left(v^{\prime}\right)\right)$, where $\widehat{\chi}: \Gamma\left(\left(\widehat{A^{*}}\right)^{*}\right) \rightarrow$ 
$L\left(\widehat{A^{*}}\right), \chi: \Gamma(A) \rightarrow L\left(A^{*}\right)$ and $\Sigma: \Gamma\left(\left(\widehat{A^{*}}\right)^{*}\right) \rightarrow \Gamma(A)$ is the map between sections induced by the homomorphism $\widetilde{\Pi}$, i.e. $\Sigma\left(v^{\prime}\right)(x)=\left(\widetilde{\Pi}_{x}\right)^{*}\left(v_{\pi(x)}^{\prime}\right)$ for all $x \in M$ and $v^{\prime} \in \Gamma\left(\left(\widehat{A^{*}}\right)^{*}\right)$. Then, $\chi(v)=\chi\left(\Sigma\left(v^{\prime}\right)\right)$, that is, $v=\Sigma\left(v^{\prime}\right)$. When $F=f \circ \tau$ is a basic function on $A^{*}$ whose differential is zero on $C$, there exists a basic function $\widehat{F}=f^{\prime} \circ \widehat{\tau}$ on $\widehat{A^{*}}$ such that, $F=\widehat{F} \circ \Pi$. Then, $f \circ \tau=f^{\prime} \circ \widehat{\tau} \circ \Pi=f^{\prime} \circ \pi \circ \tau$, that is, $f=f^{\prime} \circ \pi$.

We will show that the Lie algebroid reduction (LAR) implies the Poisson reduction $(\mathrm{PR})$ of its associated Lie co-algebroid, in the sense of MarsdenRatiu.

$$
\left(A, \rho,[\cdot, \cdot]_{A}\right) \stackrel{L A R}{\longrightarrow}\left(\widehat{A}, \widehat{\rho},[\cdot, \cdot]_{\widehat{A}}\right) \Longrightarrow\left(A^{*}, \Lambda_{A^{*}}\right) \stackrel{P R}{\longrightarrow}\left((\widehat{A})^{*}, \Lambda_{\widehat{A}^{*}}\right),
$$

But, the Poisson reduction of the Lie co-algebroid does not imply, in general, the Lie algebroid reduction, unless the reduction conditions (C1) and (C3) are satisfied.

$$
\left(A, \rho,[\cdot, \cdot]_{A}\right) \stackrel{L A R}{\longrightarrow}\left(\widehat{A}, \widehat{\rho},[\cdot, \cdot]_{\widehat{A}}\right) \Longleftrightarrow\left(A^{*}, \Lambda_{A^{*}}\right) \stackrel{P R}{\longrightarrow}\left((\widehat{A})^{*}, \Lambda_{\widehat{A}^{*}}\right)
$$

Lemma 4.1. Let $\left(A, \rho,[\cdot, \cdot]_{A}\right)$ be a reducible Lie algebroid whose reduced Lie algebroid is $\left(\widehat{A}, \widehat{\rho},[\cdot, \cdot]_{\widehat{A}}\right)$, according to theorem 3.11. Then, the Lie coalgebroid $(\widehat{A})^{*}$ is endowed with a linear Poisson bracket $\{\cdot, \cdot\}_{\wedge}$ that satisfies condition ( 7$)$.

Proof: Since the Lie algebroid $A$ is reducible, the bundle $\widehat{A}$ is endowed with a Lie algebroid structure according to the reduction theorem 3.11. Thus, the dual bundle of $\widehat{A}$ is endowed with a Poisson structure given by:

$\{f \circ \widehat{\tau}, g \circ \widehat{\tau}\}_{\wedge}=0,\left\{\widehat{\chi}\left(v^{\prime}\right), f \circ \widehat{\tau}\right\}_{\wedge}=\widehat{\rho}\left(v^{\prime}\right) f \circ \widehat{\tau},\left\{\widehat{\chi}\left(v^{\prime}\right), \widehat{\chi}\left(w^{\prime}\right)\right\}_{\wedge}=\chi\left(\left[v^{\prime}, w^{\prime}\right]_{\widehat{A}}\right)$,

where $f$ and $g$ are smooth functions on $\widehat{M}$ and $v^{\prime}$ and $w^{\prime}$ are sections of $\widehat{A}$. But $\widehat{\tau} \circ \widetilde{\Pi}=\pi \circ \tau$ and $\operatorname{Im} \Sigma$ is a subalgebra of $\Gamma(A)$, and so:

$\left\{\widehat{\chi}\left(v^{\prime}\right), f \circ \widehat{\tau}\right\}_{\wedge} \circ \widetilde{\Pi}=\widehat{\rho}\left(v^{\prime}\right) f \circ \pi \circ \tau, \quad\left\{\widehat{\chi}\left(v^{\prime}\right), \widehat{\chi}\left(w^{\prime}\right)\right\}_{\wedge} \circ \widetilde{\Pi}=\chi\left(\left[\Sigma\left(v^{\prime}\right), \Sigma\left(w^{\prime}\right)\right]_{A}\right)$.

Since $\widehat{\rho}:=\pi_{*} \circ \rho \circ \boldsymbol{\Sigma}$, we have

$$
\left\{\widehat{\chi}\left(v^{\prime}\right), f \circ \widehat{\tau}\right\}_{\wedge} \circ \widetilde{\Pi}=\rho\left(\Sigma\left(v^{\prime}\right)\right)(f \circ \pi) \circ \tau=\left\{\widehat{\chi}\left(v^{\prime}\right) \circ \widetilde{\Pi}, f \circ \pi \circ \tau\right\}_{A^{*}} .
$$

Once again by $\widehat{\tau} \circ \widetilde{\Pi}=\pi \circ \tau$, we obtain

$$
\left\{\widehat{\chi}\left(v^{\prime}\right), f \circ \widehat{\tau}\right\}_{\wedge} \circ \widetilde{\Pi}=\left\{\widehat{\chi}\left(v^{\prime}\right) \circ \widetilde{\Pi}, f \circ \widehat{\tau} \circ \widetilde{\Pi}\right\}_{A^{*}} .
$$


We also have

$$
\begin{aligned}
\left\{\widehat{\chi}\left(v^{\prime}\right), \widehat{\chi}\left(w^{\prime}\right)\right\}_{\wedge} \circ \widetilde{\Pi} & =\chi\left(\left[\boldsymbol{\Sigma}\left(v^{\prime}\right), \boldsymbol{\Sigma}\left(w^{\prime}\right)\right]_{A}\right)=\left\{\chi\left(\boldsymbol{\Sigma}\left(v^{\prime}\right)\right), \chi\left(\boldsymbol{\Sigma}\left(w^{\prime}\right)\right)\right\}_{A^{*}} \\
& =\left\{\widehat{\chi}\left(v^{\prime}\right) \circ \widetilde{\Pi}, \widehat{\chi}\left(w^{\prime}\right) \circ \widetilde{\Pi}\right\}_{A^{*}} .
\end{aligned}
$$

As the bracket of basic functions is zero, the condition (7) is trivially satisfied for this type of functions. Therefore, the condition (7) holds for all affine functions $\widehat{F}$ on $(\widehat{A})^{*}$ and extensions $\widehat{F} \circ \widetilde{\Pi}$ whose differentials belong to the annihilator of $C$. Since the set of differentials of affine functions on $(\widehat{A})^{*}$ generates the cotangent space in each point of $(\widehat{A})^{*}$, we conclude that the condition holds for all functions $\widehat{F}$ of $(\widehat{A})^{*}$.

Theorem 4.2. In the conditions of the previous lemma, let $C=\operatorname{Ker} T \widetilde{\Pi}$. Then $\left(A^{*}, C\right)$ is a reducible Poisson manifold whose reduced Poisson manifold is the Lie co-algebroid of $\widehat{A},\left((\widehat{A})^{*},\{\cdot, \cdot\}_{\wedge}\right)$.

Proof: We have to show that $C$ is a distribution in the conditions of Poisson reduction, in the sense of Marsden-Ratiu. The first condition is obvious because $C$ is the kernel of a surjective submersion. We just need to prove condition 2. Given two functions $F, G \in C^{\infty}\left(A^{*}\right)$ with $d F, d G \in C^{0}$, there exist $\widehat{F}, \widehat{G} \in C^{\infty}\left(\widehat{A^{*}}\right)$, such that, $F=\widehat{F} \circ \widetilde{\Pi}$ and $G=\widehat{G} \circ \widetilde{\Pi}$. By the above lemma, we have

$$
\{F, G\}_{A^{*}}=\{\widehat{F}, \widehat{G}\}_{\wedge} \circ \widetilde{\Pi},
$$

then $d\{F, G\}_{A^{*}} \in C^{0}$. Therefore, $\left(A^{*}, C\right)$ is a reducible Poisson manifold.

Proposition 4.3. Let $\left(A, \rho,[\cdot, \cdot]_{A}\right)$ be a Lie algebroid over $M$ and suppose that $C$ is a subbundle of $T A^{*}$ satisfying the Marsden-Ratiu reduction conditions for the Poisson structure of the Lie co-algebroid $A^{*}$. Then, the space $\operatorname{Im} \Sigma$ is a Lie subalgebra of $\Gamma(A)$.

Proof: We have to show that $\left[\Sigma\left(v^{\prime}\right), \Sigma\left(w^{\prime}\right)\right]_{A} \subset \operatorname{Im} \Sigma$ for all sections $v^{\prime}, w^{\prime} \in$ $\Gamma(\widehat{A})$. Let $F=\widehat{\chi}\left(v^{\prime}\right) \circ \widetilde{\Pi}$ and $G=\widehat{\chi}\left(w^{\prime}\right) \circ \widetilde{\Pi}$ be two linear functions in $A^{*}$ with differentials vanishing in $C$. Then,

$$
\{F, G\}_{A^{*}}=\left\{\chi\left(\Sigma\left(v^{\prime}\right)\right), \chi\left(\Sigma\left(w^{\prime}\right)\right)\right\}_{A^{*}}=\chi\left(\left[\Sigma\left(v^{\prime}\right), \Sigma\left(w^{\prime}\right)\right]_{A}\right),
$$

with $v^{\prime}, w^{\prime} \in \Gamma(\widehat{A})$. On the other hand, from condition 7 , we have

$$
\{F, G\}_{A^{*}}=\left\{\widehat{\chi}\left(v^{\prime}\right), \widehat{\chi}\left(v^{\prime}\right)\right\}_{\wedge} \circ \widetilde{\Pi} .
$$


Since the reduced bracket is linear, there exists a section $u^{\prime}$ of $\widehat{A}=\left(\widehat{A^{*}}\right)^{*}$, such that, $\{F, G\}_{A^{*}}=\chi\left(\Sigma\left(u^{\prime}\right)\right)$. Therefore,

$$
\chi\left(\left[\Sigma\left(v^{\prime}\right), \boldsymbol{\Sigma}\left(w^{\prime}\right)\right]_{A}\right)=\chi\left(\Sigma\left(u^{\prime}\right)\right) .
$$

Proposition 4.4. In the conditions of the previous proposition, $(\widehat{A}, \widehat{p}, \widehat{M})$ is endowed with a Lie algebroid structure whose anchor is given by $\widehat{\rho}=T \pi \circ \rho \circ S$ and the bracket on the sections satisfies $\boldsymbol{\Sigma}\left(\left[v^{\prime}, w^{\prime}\right]_{\widehat{A}}\right)=\left[\boldsymbol{\Sigma}\left(v^{\prime}\right), \boldsymbol{\Sigma}\left(w^{\prime}\right)\right]_{A}$ for all $v^{\prime}, w^{\prime} \in \Gamma(\widehat{A})$.

Proof: The reduced bracket is linear, then the dual bundle of $\widehat{A^{*}}$ is endowed with a Lie algebroid structure. The bracket of sections of $\widehat{A}$ is defined by the condition $\boldsymbol{\Sigma}\left(\left[v^{\prime}, w^{\prime}\right]_{\widehat{A}}\right)=\left[\boldsymbol{\Sigma}\left(v^{\prime}\right), \boldsymbol{\Sigma}\left(w^{\prime}\right)\right]_{A}$, for all $v^{\prime}, w^{\prime} \in \Gamma(\widehat{A})$. Given a linear function $F=\chi\left(v^{\prime}\right) \circ \widetilde{\Pi}$ and a basic function $G=g \circ \widehat{\tau} \circ \widetilde{\Pi}$ on $A^{*}$, then by (7),

$$
\{F, G\}_{A^{*}}=\left\{\chi\left(v^{\prime}\right), g \circ \widehat{\tau}\right\}_{\wedge} \circ \widetilde{\Pi} .
$$

By definition of $\{\cdot, \cdot\}_{A^{*}}$, we have

$\{F, G\}_{A^{*}}=\left\{\chi\left(\Sigma\left(v^{\prime}\right)\right), g \circ \pi \circ \tau\right\}_{A^{*}}=\left[T \pi \circ \rho \circ \Sigma\left(v^{\prime}\right)\right] g \circ \tau \circ \pi=\left[T \pi \circ \rho \circ \Sigma\left(v^{\prime}\right)\right] g \circ \widehat{\tau} \circ \widetilde{\Pi}$.

Since $\widetilde{\Pi}$ is a surjective submersion, by (8) we obtain

$$
\left\{\chi\left(v^{\prime}\right), g \circ \widehat{\tau}\right\}_{\wedge}=\left[T \pi \circ \rho \circ \Sigma\left(v^{\prime}\right)\right] g \circ \widehat{\tau} .
$$

By the definition $\boldsymbol{\Sigma}\left(v^{\prime}\right)(x)=S_{x}^{\pi(x)}\left(v^{\prime}(\pi(x))\right.$ for all $x \in M$, we conclude $\widehat{\rho}=T \pi \circ \rho \circ S$.

When the homomorphism $(\widetilde{\Pi}, \pi)$ satisfies the reduction conditions $(\mathrm{C} 1)$ and $(\mathrm{C} 3)$ with respect to $(\Pi, \pi):(A, p, M) \rightarrow(\widehat{A}, \widehat{p}, \widehat{M})$, the Lie algebroid $A$ is reducible.

Let us present two more examples of reduction:

Example 4.1. Let $G$ be a finite dimensional Lie group with Lie algebra $\mathfrak{g}$ and $A=T G$ the tangent bundle of $G$. The canonical lifting of the action of $G$ on itself by left translation defines a regular left action of $G$ on $A^{*}=T^{*} G$ whose space of orbits is $\mathfrak{g}^{*}$. The canonical projection $\widetilde{\Pi}: T^{*} G \rightarrow \mathfrak{g}^{*}=T^{*} G / G$ is a morphism of vector bundles over $\pi: G \rightarrow G / G=\{\cdot\}$, where the distribution $\operatorname{KerT} \widetilde{\Pi}$ is the tangent space to the orbits of $T^{*} G$. Recall that we can identify $T^{*} G$ with $G \times \mathfrak{g}^{*}$ and, with this identification, $\widetilde{\Pi}(g, \alpha)=\alpha$ for all $g \in G$ and $\alpha \in \mathfrak{g}^{*}$; $\widetilde{\Pi}$ is clearly a homomorphism of vector bundles. In theses 
conditions, $\mathfrak{g}^{*}$ is endowed with a linear Poisson structure such that $\widetilde{\Pi}$ is a Poisson morphism [20].

Let $(\Pi, \pi):(T G, p, G) \rightarrow(\mathfrak{g}, \widehat{p},\{\cdot\})$ be the canonical projection defined by the tangent representation of the action of $G$ on itself. Since $T G \equiv G \times \mathfrak{g}$ we have $\Pi(g, X)=X$ for all $X \in \mathfrak{g}$ e $g \in G$. The homomorphism $(\widetilde{\Pi}, \pi)$ satisfies the reduction conditions with respect to $(\Pi, \pi)$ and $\operatorname{Im} \Sigma$ is a Lie subalgebra of $\Gamma(T G)$. Therefore, we can conclude that $\mathfrak{g}$ is a reduced Lie algebroid of $T G$, when the tangent bundle $T G$ is endowed with the usual Lie algebroid structure $\left(i d_{T G},[\cdot, \cdot]\right)$ and $\mathfrak{g}$ with the usual Lie algebroid structure in a finite dimensional Lie algebra.

Example 4.2 (Toda). A finite non periodic Toda system is a system of $n$ particles in a line, with a exponential interaction between neighbor particles described by the Hamiltonian

$$
H\left(q_{1}, \ldots q_{n}, p_{1}, \ldots, p_{n}\right)=\frac{1}{2} \sum_{k=1}^{n} p_{k}^{2}+\sum_{k=1}^{n-1} e^{\left(q_{k-1}-q_{k}\right)},
$$

where $q_{k}$ represent the local coordinates of the configuration space $\mathbb{R}^{n}$ and $p_{k}$ the associated moments. The phase space $T^{*} \mathbb{R}^{n}$ is endowed with the canonical symplectic structure $\sigma=\sum_{i=1}^{n} d q_{i} \wedge d p_{i}$, i.e. $\left\{q_{i}, p_{i}\right\}=1$ and the other fundamental brackets are null. Let us now consider the manifold $\mathbb{R}^{2 n-1}$ with coordinates denoted $a_{1}, \ldots, a_{n-1}, b_{1}, \ldots, b_{n}$. This manifold can be endowed with a Poisson bracket defined by:

$$
\left\{a_{i}, b_{i}\right\}=-\frac{1}{4} a_{i},\left\{a_{i}, b_{i+1}\right\}=\frac{1}{4} a_{i},
$$

the other brackets are zero. The Flaschka transformation [9], defined by

$$
\widetilde{\Pi}: \begin{array}{ccc}
T^{*} \mathbb{R}^{n} & \longrightarrow & \mathbb{R}^{2 n-1} \\
\left(q_{1}, \ldots, q_{n}, p_{1}, \ldots, p_{n}\right) & \longmapsto\left(a_{1}, \ldots, a_{n-1}, b_{1}, \ldots, b_{n}\right)
\end{array}
$$

with $b_{i}=-\frac{1}{2} p_{i}$ and $a_{i}=\frac{1}{2} e^{\frac{\left(q_{k}-q_{k+1}\right)}{2}}$, is a symplectic realization of $\mathbb{R}^{2 n-1}$ with the above mentioned Poisson structure, i.e. $\widetilde{\Pi}: T^{*} \mathbb{R}^{n} \rightarrow \mathbb{R}^{2 n-1}$ is a Poisson map. Therefore, we can look at this Poisson manifold as a reduced manifold of $T^{*} \mathbb{R}^{n}$. With the identification $\mathbb{R}^{2 n-1}=\mathbb{R}^{n-1} \times \mathbb{R}^{n}$, we observe that the Flaschka transformation is a homomorphism of vector bundles over the map $\pi:\left(q_{1}, \ldots, q_{n}\right) \in \mathbb{R}^{n} \rightarrow\left(a_{1}, \ldots, a_{n-1}\right) \in \mathbb{R}^{n-1}$, where $T^{*} \mathbb{R}^{n}$ is the cotangent bundle to the manifold $\mathbb{R}^{n}$ and $\mathbb{R}^{n-1} \times \mathbb{R}^{n}$ is a trivial bundle over $\mathbb{R}^{n-1}$. Once 
the Poisson structures in the vector bundles are linear, this means that the dual bundles $T \mathbb{R}^{n}$ and $\mathbb{R}^{n-1} \times \mathbb{R}^{n}$ are endowed with Lie algebroid structures. Thus, we can ask if the usual Lie algebroid structure in $T \mathbb{R}^{n}$ is reduced into the Lie algebroid structure in $\mathbb{R}^{n-1} \times \mathbb{R}^{n}$. Actually this is the case. The projection of $T \mathbb{R}^{n} \equiv \mathbb{R}^{n} \times \mathbb{R}^{n}$ on $\mathbb{R}^{n-1} \times \mathbb{R}^{n}$ is given by the homomorphism of vector bundles $\Pi=\left(\pi,-2 \operatorname{id}_{\mathbb{R}^{n}}\right)$ over $\pi$. This homomorphism together with the Flaschka transformation satisfy the reduction conditions of Lie algebroids. Note that $\operatorname{Ker} \Pi_{x}=\{0\}$ for all $x \in \mathbb{R}^{n}$ and $\operatorname{Im} \Sigma$ is a Lie subalgebra of $\mathfrak{X}\left(\mathbb{R}^{n}\right)$ because the Flaschka transformation is a Poisson morphism.

\section{Reduction of Lie algebroids with symmetry}

In this section, we recall the definition of representation of Lie groups in vector bundles following Cariñena and al. [3] and we define representation of Lie groups in Lie algebroids in agreement with [25]. Next we prove, by showing that the Lie algebroid reduction conditions hold, that if a representation of a Lie group in a Lie algebroid defining a free and proper action on the Lie algebroid is given, then such Lie algebroid can be reduced.

5.1. Lie group representation on Lie algebroids. Let $G$ be a Lie group $G$ with neutral element $e$ and Lie algebra $\mathfrak{g}$.

Definition 5.1 ([3]). Given a vector bundle $(A, \pi, M)$, a map $\Phi: G \rightarrow$ $\operatorname{Aut}(A)$ is said to be a representation of the Lie group $G$ in the vector bundle $A$, if it satisfies the following properties:

(i) $\Phi\left(g g^{\prime}\right)=\Phi(g) \Phi\left(g^{\prime}\right)$ for all $g, g^{\prime} \in G$;

(ii) $\bar{\Phi}: G \times A \rightarrow A$, defined by $\bar{\Phi}(g, v)=\Phi(g) v$, is smooth for all $g \in G$ and $v \in A$;

Such vector bundle representation of $G$ in $A$ gives rise to a linear representation $\mathcal{R}: G \rightarrow \operatorname{Aut}(\Gamma(A))$ of $G$ in the space $\Gamma(A)$ of sections of $A$ :

$$
[\mathcal{R}(g)(v)](g x)=\Phi(g)_{x} v_{x}
$$

for each $v \in \Gamma(A)$, where $\phi_{g}: x \mapsto \phi(g, x)=g x$ for each $g \in G$ and $x \in M$.

The representations of Lie groups in vector bundles have been playing a relevant rôle in quantum mechanics. Actually, a particularly important example is that of induced representations of groups [26] which are used to obtain the classification of elementary systems [30], and the later generalization of locally operating representations of Lie transformation groups $[11,12]$. 
These are linear or multiplier representations of a connected Lie group acting transitively (on the left) on a manifold $M$, and the representation space is assumed to be the linear space of sections of a vector bundle over $M$, the relevant equivalence concept being local equivalence.

As we can see, the representation $\Phi$ defines an action $\bar{\Phi}$ of $G$ on the vector bundle $A$, with base map $\phi$, and induces a representation of the Lie group $G$ in the space of sections of $A$. We define the contragredient representation $\Phi^{c}$ (or co-action for short) of $\Phi$ as follows:

$$
\left\langle\Phi^{c}(g)(\alpha), v\right\rangle:=\left\langle\alpha, \Phi\left(g^{-1}\right)(v)\right\rangle,
$$

for each $\alpha \in A^{*}$ and $v \in A$. This co-action defines a representation of $G$ on the vector bundle $A^{*}$ that determines a linear representation $\mathcal{R}^{c}$ of $G$ in the space of sections of $A^{*}$. This linear representation is the linear contragredient representation of $\mathcal{R}$.

In what follows $\left(A, \rho,[\cdot, \cdot]_{A}\right)$ is a Lie algebroid over $M$.

Definition 5.2. A representation $\Phi: G \rightarrow \operatorname{Aut}(A)$ of the Lie group $G$ on the vector bundle $A$ is said to be a Lie algebroid representation, if for each $g \in G$ the associated linear representation $\mathcal{R}(g)$ is an automorphism of Lie algebroids, i.e.

(i) $\phi_{g *} \circ \rho=\rho \circ \mathcal{R}(g)$;

(ii) $\mathcal{R}(g)\left([v, w]_{A}\right)=[\mathcal{R}(g) v, \mathcal{R}(g) w]_{A}$, for all $v, w \in \Gamma(A)$.

In the above definition, $\phi_{g *}: \mathfrak{X}(M) \rightarrow \mathfrak{X}(M)$ is the morphism given by $\left(T \phi_{g}, \phi_{g}\right):\left(T M, \tau_{M}, M\right) \rightarrow\left(T M, \tau_{M}, M\right)$ on the spaces of sections of the tangent bundle $T M$, i.e. $\phi_{g *}(X)=T \phi_{g} \circ X \circ \phi_{g^{-1}}$ for all $X \in \mathfrak{X}(M)$. Note that the first condition of the definition is equivalent to

$$
\rho=\phi_{g^{-1} *} \circ \rho \circ \mathcal{R}(g),
$$

where $\phi_{g^{-1}}$ is the inverse map of $\phi_{g}$.

Example 5.1. Let $\mathfrak{g}$ be a Lie algebra of finite dimension endowed with its usual Lie algebroid structure. The adjoint representation of the Lie group $G$ on $\mathfrak{g}$, defines a Lie algebroid representation $A d: G \rightarrow G L(\mathfrak{g} ; \mathbb{R})$. Note that in this situation the group representation on the vector bundle $\mathfrak{g}$ coincide with the linear representation of the group on the space of sections.

We next prove, using the definition of Lie algebroid representation, that the contragredient representation of $\Phi$ defines, for each $g \in G$, a Poisson morphism and, therefore, the associated action is a Poisson action: 
Proposition 5.3. Given a representation $\Phi: G \rightarrow \operatorname{Aut}(A)$ of the Lie group $G$ on the Lie algebroid $A$, the contragredient representation $\Psi=\Phi^{c}: G \rightarrow$ Aut $\left(A^{*}\right)$ defines a Poisson action of the Lie group $G$ on the Lie co-algebroid $\left(A^{*}, \Lambda_{A^{*}}\right)$.

Proof: We have to show that $\Psi(g): A^{*} \rightarrow A^{*}$ is a Poisson morphism for all $g \in G$. The affine functions generate the cotangent space to $A^{*}$, and then it is sufficient to show that

$$
\{F \circ \Psi(g), G \circ \Psi(g)\}_{A^{*}}=\{F, G\}_{A^{*}} \circ \Psi(g)
$$

for all affine functions $F$ and $G$ on $A^{*}$. Since the Poisson bracket on the Lie co-algebroid $A^{*}$ is zero on the basic functions, we only need to show the above equality when both functions are linear functions or when one is linear and the other is basic. Let $F=\chi(v)$ and $G=\chi(w)$ be two linear functions on $A^{*}$ associated, respectively, to the sections $v$ and $w$ of $A$. Taking into account that $F \circ \Psi(g)=\chi(v) \circ \Psi(g)=\chi\left(\mathcal{R}\left(g^{-1}\right)(v)\right)$, and using the definition of the bracket $\{\cdot, \cdot\}_{A^{*}}$, we have

$$
\begin{aligned}
\{F \circ \Psi(g), G \circ \Psi(g)\}_{A^{*}} & =\left\{\chi\left(\mathcal{R}\left(g^{-1}\right)(v)\right), \chi\left(\mathcal{R}\left(g^{-1}\right)(w)\right)\right\}_{A^{*}} \\
& =\chi\left(\left[\mathcal{R}\left(g^{-1}\right)(v), \mathcal{R}\left(g^{-1}\right)(w)\right]_{A}\right) .
\end{aligned}
$$

But, for each $g \in G$, the linear representation $\mathcal{R}$ is a homomorphism of Lie algebras; then,

$$
\begin{aligned}
\{F \circ \Psi(g), G \circ \Psi(g)\}_{A^{*}} & =\chi\left(\mathcal{R}\left(g^{-1}\right)\left([v, w]_{A}\right)\right)=\chi\left([v, w]_{A}\right) \circ \Psi(g) \\
& =\{\chi(v), \chi(w)\}_{A^{*}} \circ \Psi(g)=\{F, G\}_{A^{*}} \circ \Psi(g) .
\end{aligned}
$$

Now, let $F=\chi(v)$ be the linear function on $A^{*}$ associated to the section $v$ of $A$ and let $G=h \circ \tau$ be a basic function on $A^{*}$. Then,

$$
\begin{aligned}
\{F \circ \Psi(g), G \circ \Psi(g)\}_{A^{*}} & =\left\{\chi\left(\mathcal{R}\left(g^{-1}\right)(v)\right), h \circ \tau \circ \Psi(g)\right\}_{A^{*}} \\
& =\left\{\chi\left(\mathcal{R}\left(g^{-1}\right)(v)\right), h \circ \phi_{g} \circ \tau\right\}_{A^{*}} \\
& =\rho\left(\mathcal{R}\left(g^{-1}\right)(v)\right)\left(h \circ \phi_{g}\right) \circ \tau \\
& =\left(\left(\phi_{g}\right)_{*} \circ \rho \circ \mathcal{R}\left(g^{-1}\right)(v)\right) h \circ \phi_{g} \circ \tau \\
& \stackrel{(9)}{=} \rho(v) h \circ \phi_{g} \circ \tau=\rho(v) h \circ \tau \circ \Psi(g) \\
& =\{\chi(v), h \circ \tau\}_{A^{*}} \circ \Psi(g)=\{F, G\}_{A^{*}} \circ \Psi(g) .
\end{aligned}
$$


On the other hand, given a representation $\Psi$ of $G$ on the Lie co-algebroid $A^{*}$ whose associated action $\bar{\Psi}$ is Poisson, one can easily prove that the contragredient representation of $G$ in $A$ is a Lie algebroid representation.

Example 5.2. Let us consider the cotangent bundle $T^{*} M$ endowed with its usual symplectic structure and suppose that $\phi$ is a left action of the Lie group $G$ on $M$. The canonical lifting of this Lie group action is an action on $T^{*} M$,

$$
\begin{aligned}
\Psi: G \times T^{*} M & \rightarrow T^{*} M \\
(g, \alpha) & \mapsto \Psi(g, \alpha)={ }^{t} \phi_{g}(\alpha)
\end{aligned}
$$

where ${ }^{t} \phi_{g}: T^{*} M \rightarrow T^{*} M$ is the canonical lifting of the diffeomorphism $\phi_{g}$ : $M \rightarrow M$

$$
\left\langle{ }^{t} \phi_{g}(\alpha), v\right\rangle=\left\langle\alpha, T \phi_{g^{-1}}(v)\right\rangle .
$$

Note that ${ }^{t} \phi_{g}=\left(T \phi_{g^{-1}}\right)^{*}=:(T \phi)^{c}(g)$. The contragredient representation of the representation defined by the action $\Psi$ is a map $T \phi: G \rightarrow \operatorname{Aut}(T M)$ given by $(T \phi)(g)=T \phi_{g}$ for all $g \in G$. It is a representation of $G$ on the vector bundle $T M$. The linear representation associated to $T \phi$ is given by $\mathcal{R}(g)=\phi_{g *}$.

Let us suppose that TM is endowed with its usual Lie algebroid structure. Since the action $\Psi$ is symplectic, the contragredient representation $T \phi$ defines a Lie algebroid representation. Note that the first condition of the definition is satisfied because the anchor is the identity map and $\mathcal{R}(g)=\phi_{g *}$, and the second condition also holds because the map $\Psi_{g^{-1}}$ is a Poisson morphism of the Lie co-algebroid $T^{*} M$ for all $g \in G$.

5.2. Reduction. Let us consider a Lie algebroid $\left(A, \rho,[\cdot, \cdot]_{A}\right)$ over $M$ and a Lie algebroid representation $\Phi$ of the Lie group $G$ on $A$. Let $\Psi=\Phi^{c}$ be the contragredient representation of $G$ on $A^{*}$. Suppose, additionally, that $\Phi$ and $\Psi$ define free and proper actions of $G$ on $A$ and $A^{*}$, respectively. Then, the canonical projection $\widetilde{\Pi}: A^{*} \rightarrow \widehat{A^{*}}=A^{*} / G$ is a surjective submersion, the projection $\widetilde{\Pi}$ is given by

$$
\widetilde{\Pi}(\alpha)=[\alpha]_{G}=\{g . \alpha:=\Psi(g) \alpha \mid g \in G\} .
$$

Since $A^{*}$ is a vector bundle, there exists a unique vector bundle structure in $\widehat{A^{*}}, \widehat{\tau}: \widehat{A^{*}} \rightarrow \widehat{M}$ such that $\widetilde{\Pi}$ is a submersion of vector bundles over the basis 
$\pi: M \rightarrow \widehat{M}$, where $\pi$ is the canonical projection of $M$ in $\widehat{M}=M / G,[19]$.

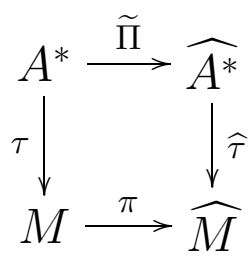

The projection $\widehat{\tau}$ is given by $\widehat{\tau}\left([\alpha]_{G}\right)=\pi(\tau(\alpha))$ for each $\alpha \in A^{*}$, that is, given an element $\alpha_{x} \in A_{x}, \widehat{\tau}\left(\left[\alpha_{x}\right]_{G}\right):=\pi\left(\tau\left(\alpha_{x}\right)\right)=\pi(x)=\widehat{x}$. The set $\widehat{A}^{*} \widehat{x}=\widehat{\tau}^{-1}(\widehat{x})$ is a vector space for all $\widehat{x} \in \widehat{M}$. Moreover, $\widetilde{\Pi}_{x}: A_{x}^{*} \rightarrow \widehat{A}^{*} \widehat{x}$ is an isomorphism of vector spaces for each $x \in M$ : if $\left[\alpha_{x}\right]_{G}=\left[\beta_{x}\right]_{G}$, there exists $g \in G$ such that $\alpha=g \beta$. Then, $x=g x$, which implies $g=e$ (because $\psi: G \times M \rightarrow M$, the base map to the action defined by $\Psi$, is a free action); therefore, $\alpha_{x}=\beta_{x}$.

In a similar way, using the canonical projection of $A$ over $A / G, \Pi: A \rightarrow$ $\widehat{A}=A / G$, we build a homomorphism of fiber bundles over $\pi: M \rightarrow \widehat{M}=$ $M / G$.

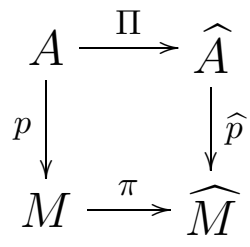

We will show that the homomorphism $(\widetilde{\Pi}, \pi)$ verifies the Lie algebroid reduction conditions. First of all, remember that the set of sections of $\widehat{A}=$ $A / G$ is isomorphic to the set of $G$-invariant sections of $A$ : if $v \in \Gamma^{G}(A)$ then $v^{\prime}=[v]_{G}$ verifies $\Pi \circ v=v^{\prime} \circ \pi$; therefore, $v^{\prime}$ is a section of $A / G$. On the other hand, if $v^{\prime} \in \Gamma(A / G)$ we have that $v(x)=\left(\Pi_{x}\right)^{-1}\left(v_{\pi(x)}^{\prime}\right)$ is a $G$-invariant section of $A[19]$.

Proposition 5.4. In the above conditions, the reduction condition (C1) holds.

Proof: Given a section $v^{\prime}$ of $A / G$, there exists a unique section $v \in \Gamma^{G}(A)$ such that $\Pi_{x}\left(v_{x}\right)=v_{\pi(x)}^{\prime}$. Thus, for $\alpha \in \Gamma(A)$ and $x \in M$, we have

$$
\left\langle\alpha_{x},\left(\widetilde{\Pi}_{x}\right)^{*}\left(v_{\pi(x)}^{\prime}\right)\right\rangle:=\left\langle\widetilde{\Pi}_{x}\left(\alpha_{x}\right), v_{\pi(x)}^{\prime}\right\rangle=\left\langle\left[\alpha_{x}\right]_{G}, v_{\pi(x)}^{\prime}\right\rangle=\left\langle\alpha_{\pi(x)}^{\prime}, v_{\pi(x)}^{\prime}\right\rangle
$$


with $\alpha^{\prime} \in \Gamma\left(A^{*} / G\right)$. The above function is $G$-invariant and linear, therefore there exists $\bar{v} \in \Gamma^{G}(A)$ such that

$$
\left\langle\alpha_{x}, \bar{v}_{x}\right\rangle=\left\langle\alpha_{\pi(x)}^{\prime}, v_{\pi(x)}^{\prime}\right\rangle=\left\langle\alpha_{x},\left(\widetilde{\Pi}_{x}\right)^{*}\left(v_{\pi(x)}^{\prime}\right)\right\rangle,
$$

and so $\left(\widetilde{\Pi}_{x}\right)^{*}\left(v_{\pi(x)}^{\prime}\right)=\bar{v}_{x}$. For all $\alpha_{x}=\left(\Pi_{x}\right)^{*}\left(\alpha_{\pi(x)}^{\prime}\right)$, we have

$$
\left\langle\alpha_{\pi(x)}^{\prime}, v_{\pi(x)}^{\prime}\right\rangle=\left\langle\alpha_{x}, \bar{v}_{x}\right\rangle=\left\langle\alpha_{\pi(x)}^{\prime}, \Pi_{x}\left(\bar{v}_{x}\right)\right\rangle
$$

then $v_{\pi(x)}^{\prime}=\Pi_{x}\left(\bar{v}_{x}\right)$. Therefore, $\left(\widetilde{\Pi}_{x}\right)^{*}\left(v_{\pi(x)}^{\prime}\right)=\bar{v}_{x}=v_{x}$. In this way,

$$
\Pi_{x} \circ S_{x}^{\pi(x)}\left(v_{\pi(x)}^{\prime}\right)=\Pi_{x} \circ\left(\widetilde{\Pi}_{x}\right)^{*}\left(v_{\pi(x)}^{\prime}\right)=\Pi_{x}\left(v_{x}\right)=\left[v_{x}\right]_{G}=v_{\pi(x)}^{\prime}
$$

for all $x \in M$.

Proposition 5.5. In the above conditions, the reduction condition (C2) holds.

Proof: We will show that $\operatorname{Im} \Sigma$ is a subalgebra of the space of sections of $A$. In fact, the image by $\Sigma$ of a section of $A / G$ is a $G$-invariant section of $A$, because $\Pi \circ \boldsymbol{\Sigma}\left(v^{\prime}\right)=v^{\prime} \circ \pi$ for all $v^{\prime} \in \Gamma(A / G)$, so

$$
v(x)=\left(\Pi_{x}\right)^{-1}\left(v_{\pi(x)}^{\prime}\right)=\Sigma\left(v^{\prime}\right)(x) .
$$

Therefore, each $G$-invariant section is of the form $\Sigma\left(v^{\prime}\right)$, with $v^{\prime} \in \Gamma(A / G)$. Taking into account that the bracket of $G$-invariant sections is still $G$-invariant, for all $v^{\prime}, w^{\prime} \in \Gamma(A / G)$, there exists a $z^{\prime} \in \Gamma(A / G)$ such that $\Pi \circ\left[\boldsymbol{\Sigma}\left(v^{\prime}\right), \boldsymbol{\Sigma}\left(w^{\prime}\right)\right]$ $=z^{\prime} \circ \pi=\Pi \circ \boldsymbol{\Sigma}\left(z^{\prime}\right)$, therefore, $\left[\boldsymbol{\Sigma}\left(v^{\prime}\right), \boldsymbol{\Sigma}\left(w^{\prime}\right)\right]=\boldsymbol{\Sigma}\left(z^{\prime}\right)$.

Each section $v$ of $A$ is related with a $G$-invariant section $v^{G}$ of $A$ and so each section of $A$ is of the form $v=\mathcal{R}(g)\left(v^{G}\right)=\mathcal{R}(g)\left(\boldsymbol{\Sigma}\left(v^{\prime}\right)\right)$, with $g \in G$ and $v^{\prime} \in \Gamma(A / G)$.

Proposition 5.6. Under the above conditions, $\Pi$ is a homomorphism of Lie algebroids and $\left(\widehat{A}, \widehat{\rho},[\cdot, \cdot]_{\widehat{A}}\right)$ is a reduced Lie algebroid of $A$.

Proof: Let $v$ be a section of Ker $\Pi$. Then,

$$
\Pi \circ v=s_{0}^{\prime} \circ \pi=\Pi \circ \Sigma\left(s_{0}^{\prime}\right),
$$

where $s_{0}^{\prime}$ is the zero section of $\widehat{A}$. Thus, there exists $g \in G$ such that $v=$ $\mathcal{R}(g)\left(\boldsymbol{\Sigma}\left(s_{0}^{\prime}\right)\right)$. But,

$$
\boldsymbol{\Sigma}\left(s_{0}^{\prime}\right)(x)=S_{x}^{\pi(x)}\left(s_{0}^{\prime}(\pi(x))\right)=0
$$


for all $x \in M$, therefore, $\boldsymbol{\Sigma}\left(s_{0}^{\prime}\right)$ is the zero section of $A, s_{0}$. Since $\mathcal{R}(g)$ is an isomorphism of the spaces of sections, we have $\mathcal{R}(g)\left(s_{0}\right)=s_{0}$. Thus, $v=s_{0}$, that is, $\Gamma(\operatorname{Ker} \Pi)=\left\{s_{0}\right\}$. From proposition 3.9, $\Pi$ is a homomorphism of Lie algebroids.

Once the reduction conditions (C1), (C2) and (C3) hold, the quotient bundle $\widehat{A}=A / G$ is endowed with a Lie algebroid structure given by the reduction theorem.

From theorem 4.2 , we know that $\left(A^{*}, C=\operatorname{Ker} \widetilde{\Pi}\right)$ is a reducible Poisson manifold. We define in $(\widehat{A})^{*}=A^{*} / G=\widehat{A^{*}}$ a linear Poisson structure $\{\cdot, \cdot\}_{\wedge}$ that verifies

$$
\{f \circ \widetilde{\Pi}, g \circ \widetilde{\Pi}\}_{A^{*}}=\{f, g\}_{\wedge} \circ \widetilde{\Pi},
$$

where $f, g \in C^{\infty}\left(A^{*} / G\right) ; F=f \circ \widetilde{\Pi}$ and $G=g \circ \widetilde{\Pi}$ are smooth functions on $A^{*}, G$-invariant extensions of $f$ and $g$, respectively. So, the Poisson bracket of $G$-invariant functions on $A^{*}$ is still $G$-invariant.

Example 5.3 (Principal bundle). Let us consider a principal bundle $P(M, G, \pi)$ over a manifold $M$ with structural group $G$. The right action of $G$ on $P$ provides a right action of $G$ on the tangent bundle $(T P, p, P)$. This action induces in the quotient space $T P / G$, a vector bundle structure over $M$, in such a way that the natural projection $\Pi: T P \rightarrow T P / G$ is a surjective submersion and a morphism of vector bundles over $\pi: P \rightarrow M$, that makes the following diagram commutative:

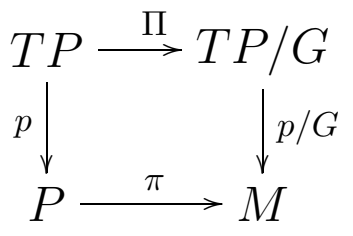

where $p / G: T P / G \rightarrow M$ is the projection given by $p / G\left([X]_{G}\right)=\pi(p(X))=$ $[p(X)]_{G}$. In the same way, we can say that the right action of $G$ on $P$ gives an action of $G$ on $\left(T^{*} P, \tau, P\right)$ that induces in the space $T^{*} P / G$ a structure of vector bundle over $M$, such that the natural projection $(\widetilde{\Pi}, \pi):\left(T^{*} P, \tau, P\right) \rightarrow$ $\left(T^{*} P / G, \tau / G, M\right)$ is a surjective submersion of vector bundles that verifies $\tau / G \circ \widetilde{\Pi}=\pi \circ \tau$, where $\tau / G: T^{*} P / G \rightarrow M$ is the projection given by $\tau / G\left([\alpha]_{G}\right)=\pi(\tau(\alpha))=[\tau(\alpha)]_{G}$. For all $\widehat{X} \in \Gamma(T P / G)$ and $u \in P$, we have

$$
\Sigma(\widehat{X})(u)=S_{u}^{\pi(u)}\left(\widehat{X}_{\pi(u)}\right)=\left(\widetilde{\Pi}_{u}\right)^{*}\left(\widehat{X}_{\pi(u)}\right)=\left(\Pi_{u}\right)^{-1}\left(\widehat{X}_{\pi(u)}\right)=X(u)
$$


with $X \in \Gamma^{G}(T P)$. Under these conditions, the vector bundle $T P / G$ is endowed with the following structure of reduced Lie algebroid:

(i) $\widehat{\rho}=T \pi \circ S$

(ii) $[\widehat{X}, \widehat{Y}]_{T P / G} \circ \pi=\Pi \circ[\boldsymbol{\Sigma}(\widehat{X}), \boldsymbol{\Sigma}(\widehat{Y})]_{T P}$ for all $\widehat{X}, \widehat{Y} \in \Gamma(T P / G)$, where $\boldsymbol{\Sigma}$ is the map between sections given by $\widetilde{\Pi}$.

The above structure is precisely the structure of gauge algebroid in $T P / G$.

\section{References}

[1] A. Cannas da Silva and A. Weinstein, Lectures on geometrical models for noncommutative algebra, University of California at Berkeley, 1998.

[2] F. Cantrijn, J.F. Cariñena and M. Crampin, Reduction of degenerate Lagrangians systems, J. Geom. Phys. 3 (3) (1986) 353-400.

[3] J.F. Cariñena, M.A. del Olmo and M. Santander, Locally operating realizations of transformation Lie Groups, J. Maths. Phys. 26 (9) (1985) 2096-2106; M. Asorey, J.F. Cariñena and M.A. del Olmo, Vector Bundle representations of groups in quantum physics, J. Phys. A: Math. Gen. 16 (1983) 1603-1609.

[4] J.F. Cariñena and H. Figueroa, Hamiltonian versus Lagrangian formulations of supermechanics, J. Phys. A: Math. Gen. 30 (1997) 2705-2724.

[5] J.F. Cariñena, J. Grabowski and G. Marmo, Contractions: Nijenhuis and Saletan tensors for general algebraic structures, J. Phys. A: Math. Gen. 34 (2001) 3769-3789.

[6] C. Chevalley and S. Eilenberg, Cohomology theory of Lie groups and Lie algebras, Trans. Amer. Math. Soc. 63 (1948) 85-124.

[7] T.J. Courant, Tangent Lie algebroids, J. Phys. A: Math. Gen. 27 (1994) 4527-4536.

[8] H. Figueroa, Variedades graduadas y sus aplicaciones en supermecánica, Tesis doctoral, Universidad de Zaragoza, Departamento de Física Teórica, 1996.

[9] H. Flaschka, The Toda lattice. II. Existence of integrals. Phys. Rev. B 9 (1974) 1924-1925.

[10] P.J. Higgins and K. Mackenzie, Algebraic Constructions in the Category of Lie Algebroids, J. Algebra 129 (1990) 194-230.

[11] H. Hoogland, On local and global equivalence of wave functions. Superequivalence of group exponents applied in Quantum Mechanics, Nuovo Cim. B 32 (1976) 427-456.

[12] H. Hoogland, Gauge equivalence of representations of symmetry groups, J. Phys. A 11 (1978) $1557-1568$.

[13] Y. Kosmann-Schwarzbach and F. Magri, Poisson-Nijenhuis structures, Ann. Inst. H. Poincaré, Phys. Théor. 53 (1990) 35-81.

[14] J.-L. Koszul, Crochet de Schouten-Nijenhuis et cohomologie, in Élie Cartan et les mathématiques d'aujourd'hui, Astérique, numéro hors série (1985) 257-271.

[15] D.A. Leites, Introduction to theory of supermanifolds, Russian Math. Surveys 35 (1) (1980) $1-64$.

[16] M. León, J.C. Marrero and E. Martínez, Lagrangian submanifolds and dynamics on Lie algebroids, J. Phys. A: Math. Gen. 38 (2005) 241-308.

[17] P. Libermann, Lie Algebroids and Mechanics. Archivum Mathematicorum (Brno) 32 (1996) $147-162$.

[18] K. Mackenzie, Lie algebroids and Lie pseudoalgebras, Bull. London Mat. Soc. 27 (1995) 97-147.

[19] K. Mackenzie, Lie groupoids and Lie algebroids in differential geometry, London Mathematical Society lecture Note Serie. 124, Cambridge University Press, 1987. 
[20] J.E. Marsden and T. Ratiu, Reduction of Poisson Manifolds, Lett. Math. Phys. 11 (1986) 161-169.

[21] E. Martínez, Lagrangian Mechanics on Lie algebroids, Acta Appl. Math. 67 (2001) 295-320.

[22] A. Meucci, Compatible Lie algebroids and the periodic Toda Lattice, J. Geom. Phys. 35 (2000) $273-287$.

[23] A. Meucci, Toda Equations, bi-Hamiltonian Systems and Compatible Lie Algebroids, Math. Phys. Anal. Geom. 4 (2001) 131-146.

[24] J. Pradines, Théorie de Lie pour les groupoïdes différentiables. Calcul différentiel dans la catégorie des groupoïdes infinitésimaux, C.R. Acad. Sci. Paris Sér. A 264 (1967) 245-248.

[25] M. Rodríguez-Olmos, Formalismo Lagrangiano en Algebroides de Lie: Reducción Lagrangiana.

[26] D.J. Simms, Lie groups and Quantum Mechanics, Lecture Notes in Mathematics 52 (Berlín: Springer-Verlag), 1968.

[27] A. Vaintrob, Lie Algebroids and homological vector fields, Rus. Math. Surv. 52 (1997) 428-429.

[28] F. Warner, Foundations of Differential Manifolds and Lie Groups, Springer-Verlag, 1980.

[29] A. Weinstein, Lagrangian Mechanics and Groupoids, in the book: Mechanics Day, Shadwick W.F. Krishnaprasad P.S. and Ratiu T.S. eds., American Mathematical Society, Fields Inst. Comm. 7 (1996) 207-237.

[30] E.P. Wigner, On Unitary Representations of the Inhomogeneous Lorentz Group, Ann. Math. 40 (1939) 149-204.

J.F. CARIÑNENA

Departamento de Física Teórica, Universidad de Zaragoza, 50009 Zaragoza, Spain

E-mail address: jfc@unizar.es

J.M. Nunes DA COSTA

Departamento de Matemática, Universidade de Coimbra, 3001-454 Coimbra, Portugal

E-mail address: jmcosta@mat.uc.pt

PATRÍCIA SANTOS

Departamento de Física e Matemática, Instituto Superior de Engenharia de Coimbra 3030-199 Coimbra, Portugal

E-mail address: patricia@isec.pt 